La Revue

des Droits

de l'Homme

\section{La Revue des droits de l'homme}

Revue du Centre de recherches et d'études sur les droits fondamentaux

16 | 2019

Revue des droits de l'homme - $\mathrm{N}^{\circ} 16$

\title{
Le gouvernement par standards de certification consentement et plaintes des communautés affectées
}

\section{Emmanuelle Cheyns et Laurent Thévenot}

\section{(2) OpenEdition}

\section{Journals}

Édition électronique

URL : http://journals.openedition.org/revdh/6843

DOI : $10.4000 /$ revdh.6843

ISSN : 2264-119X

Éditeur

Centre de recherches et d'études sur les droits fondamentaux

Référence électronique

Emmanuelle Cheyns et Laurent Thévenot, « Le gouvernement par standards de certification consentement et plaintes des communautés affectées », La Revue des droits de l'homme [En ligne], 16। 2019, mis en ligne le 01 juillet 2019, consulté le 09 juillet 2020. URL : http://journals.openedition.org/ revdh/6843; DOI : https://doi.org/10.4000/revdh.6843

Ce document a été généré automatiquement le 9 juillet 2020.

Tous droits réservés 


\title{
Le gouvernement par standards de certification consentement et plaintes des communautés affectées
}

\author{
Emmanuelle Cheyns et Laurent Thévenot
}

\section{NOTE DE L'AUTEUR}

Les enquêtes exposées dans ce papier ont été financées par l'Agence nationale de la recherche (ANR), sur le contrat ANR-11-CEPL-0009 (projet PRIGOUE).

1 Parmi les instruments normatifs contemporains de régulation, les standards privés et volontaires occupent une place croissante au côté des législations nationales et du droit international, prétendant suppléer à leurs déficiences. Cette montée en puissance des standards s'est accompagnée d'un déplacement de la responsabilité normalisatrice depuis les Etats nations vers des entités internationales telle que l'Organisation internationale de normalisation (ISO), ou supranationales telles que le Comité européen de normalisation (CEN), puis vers des organisations privées aucunement liées à des Etats et constituées notamment à partir de filières et chaînes de valeur transnationales répondant à des enjeux économiques considérables, telles que la multi-stakeholder " Roundtable for Sustainable Palm Oil » (RSPO) à laquelle cet article est consacré.

Une première partie introduit les enquêtes et le cadre d'analyse développé pour traiter, non de la simple extension de standards, mais d'un véritable mode nouveau de gouvernement en quête de légitimité. L'analyse porte sur le modèle politique normatif sous-jacent aux initiatives multi-parties prenantes (Multi-Stakeholder Initatives), ainsi que sur une exigence démocratique majeure avancée par des ONG : le «Free, Prior and Informed Consent ». Une deuxième partie met en regard des pouvoirs, fonctions et principes idéaux d'un État de droit national ceux qui sont assumés par le "gouvernement RSPO ». La troisième partie est centrée sur les questions soulevées par le pluralisme des registres normatifs et évaluatifs confrontés dans ce gouvernement. 
Elle traite des façons dont les préoccupations des parties prenantes reconnues comme les plus vulnérables (les " petits planteurs » et les communautés locales) doivent être transformées pour être recevables dans le standard RSPO et son gouvernement, qu'il s'agisse d'expressions de leur accord pour l'usage de terres par des sociétés, de demandes ou de plaintes qu'elles déposent.

\section{Un gouvernement par les standards en quête de légitimité : constructions politiques sous-jacentes}

3 Contrastant avec les standards de compatibilité industrielle qui furent historiquement premiers et continuent à véhiculer la fonction simple d'uniformisation, de nouveaux standards de certification se développent autour de la qualité diversifiée de produits mis sur le marché, équipant une nouvelle «économie morale (Busch 2000, 2011). Alors que le bien garanti par les premiers n'est que celui d'une efficacité d'ordre industriel $^{1}$ qu'augmente la compatibilité des produits, les standards en sont venus à soutenir les prérogatives d'Etats-nations qui ont à garantir un bien civique. Par des labels attribués à des produits et des services, les standards les qualifient pour des biens fondamentaux tels que sécurité, santé, protection de l'environnement, équité, respect de la personne et lutte contre les discriminations. Le "marché aux normes » (Thévenot 1995), qui en résulte et constitue l'ossature de ce que recouvre la notion souvent imprécise de néo-libéralisme, a acquis une portée politique et morale sans précédent. En réponse aux critiques sur son déficit d'une légitimité démocratique qui devrait être à la hauteur d'une telle ambition de " gouvernement par les standards » (Ponte Gibbon \& Vestergaard 2011, Thévenot 1997, 2009), de nouveaux dispositifs ont été mis en place. Nous les considérons dans ce chapitre, en suivant leurs principes et leur mise en pratique effective.

\subsection{Un cumul d'enquêtes rendu possible par un cadre d'analyse cohérent}

4 Afin de comprendre le fonctionnement pratique de ce nouveau mode de gouvernement transnational en quête de légitimité, nous disposons d'une série intégrée d'enquêtes et recherches qui, depuis plusieurs années, ont été menées dans un même cadre d'analyse $^{2}$. Elles ont permis d'observer ce type de standards de certification "soutenable" ayant une vocation transnationale et l'ambition de suppléer à la déficience des Etats-nations et de leurs systèmes juridiques. Créés pour gouverner des chaînes de valeur agro-industrielles multinationales, ces standards contournent à la fois les représentants de la communauté scientifique et ceux des Etats-nations (Ponte \& Cheyns 2013). Ils concernent aujourd'hui un large ensemble de filières agroindustrielles mondiales majeures : café, soja, sucre de canne, coton, fleurs, aquaculture, bœuf, biocarburant. Nos enquêtes portent sur celle de l'huile de palme, sur le standard de certification de l'«huile de palme soutenable» et son gouvernement: RSPO (Roundtable on Sustainable Palm Oil).

5 Dans notre approche, le politique est abordé à partir des opérations pratiques de mise en commun et en différend et des contraintes normatives pragmatiques pesant sur elles. Les mises en forme conventionnelles que ces opérations requièrent et les pouvoirs de coordination qu'elles confèrent sont au centre de l'analyse. Pour 
caractériser des "grammaires " normatives publiques du commun en différend, ou "commun au pluriel», nous identifions les transformations pratiques qu'elles demandent aux acteurs pour qu'ils fassent entendre leurs voix différentes dans le public auquel ils prennent part. Afin de mettre en lumière ces transformations souvent sous-estimées, nous suivons les acteurs depuis leurs lieux d'habitation, attachements et relations avec la nature environnante. Nous utilisons une grille d'analyse qui distingue des relations mises en valeur entre les personnes et leur environnement - engagements depuis l'engagement familier très personnalisé jusqu'à des engagements publiquement justifiés par référence au bien commun (Thévenot 2006, 2014).

6 Notre analyse part de l'engagement familier dans un espace cultivé et habité, engagement des plus personnels pour des biens intimes, personnels et locaux, que les personnes apprécient selon un format évaluatif et normatif très éloigné de ceux qu'exige le public. Les dispositifs publics de participation pour faire entendre ces maux se montrent fort contraignants quant aux «formats de participation» (Richard-Ferroudji 2011) acceptables. Ils disqualifient des biens et formats d'évaluation d'acteurs les plus faibles (Charles 2012, 2015). Pour étudier la manière dont ce gouvernement prend en compte des biens et droits, notre investigation a suivi les personnes les plus vulnérables (les «petits planteurs» et les communautés locales riveraines, affectées par le développement de plantations industrielles, en Indonésie), depuis leur village reculé jusqu'à la " convention » annuelle de la roundtable qui se déroule dans un grand hôtel international d'une métropole régionale. Elle a mis en évidence la chaîne des transformations que leurs préoccupations doivent subir pour qu'ils fassent entendre leurs griefs, ainsi que le concours de nombreuses ONG à cette mise en forme conventionnelle.

\subsection{L'assise de la grammaire libérale des intérêts et la modalité multistakeholder de composition d'un commun}

7 Les grammaires du commun et du différend se distinguent par les transformations qu'elles imposent aux préoccupations personnelles pour que celles-ci soient exprimables en public. La grammaire des "grandeurs» de bien commun (Boltanski \& Thévenot 1987, 1991) demande que l'expression soit qualifiée par une conception du bien commun, ce qui requiert une transformation considérable. La grammaire "libérale » des intérêts (Thévenot 2008, 2015a) est moins exigeante: les engagements familiers doivent être transformés, non directement dans une qualification de bien commun mais dans une qualification d'intérêt («stake») pour lequel la personne choisit de prendre publiquement parti, la coalition de ces intérêts constituant le bien commun de la communauté. Il ne faut cependant pas sous-estimer le travail requis pour exprimer ses attachements intimes en termes de choix individuels entre des options "opinions ", "préférence ", « intérêt » - exposées dans un format communément identifiable par les autres individus. En particulier, ces options, entre lesquelles l'individu choisit, doivent se présenter à tous les autres participants du public libéral comme autant de "plans d'action" dans lesquels ils sont susceptibles de se projeter volontairement. Selon cette grammaire, le format d'engagement privilégié à tous les autres est celui de l'engagement en plan - ou projet - que présuppose la visée individuelle et volontaire d'un objectif limité. L'expression du différend présuppose la réduction des évaluations à des préférences individuelles pour de tels plans optionnels. C'est par une négociation entre de tels plans que se compose le bien de la communauté. Les biens 
communs en dispute dans la grammaire des grandeurs, tels que la solidarité de grandeur civique requise dans la lutte contre des inégalités, tout autant que les biens du proche qui touchent au plus près la personne tels que les attachements familiers aux (mi)lieux et à leurs usages, s'en trouvent disqualifiés (Cheyns 2011).

8 Notre cadre d'analyse permet ainsi de mettre en lumière des types d'oppression qui ne se limitent pas aux exclusions de participants. Il montre en outre que les notions de liberté, ou d'horizontalité, souvent mises en avant pour caractériser le public libéral, sont trompeuses car elles méconnaissent ces contraintes de transformation ainsi que la position hiérarchique supérieure d'une voix qui s'expose dans le format public du choix autonome individuel au regard d'expressions disqualifiées parce que trop intimes ou particulières («self-centered », « narcissist »).

9 Selon une variation courante de la grammaire libérale, les intérêts ne sont pas seulement ceux d'un individu mais d'un ensemble d'individus (Eranti 2008, Luhtakallio \& Thévenot 2018, Moody \& Thévenot 2000). Ils ne prétendent pas pour autant contribuer directement au bien commun, mais doivent pour se faire former "alliance " avec d'autres intérêts au sein d'une « coalition ». Dans le dispositif dit «multistakeholder » qui gouverne le standard d'huile de palme soutenable, les différents intérêts collectifs (stakes) sont explicitement rapportés à sept catégories de stakeholders en principe dotés d'une égale voix individuelle. Cette qualification fixée risque de rompre l'égalité entre les voix, que requiert formellement la matrice libérale ${ }^{3}$.

\subsection{Une exigence démocratique avancée par des ONG, le « Free Prior Informed Consent [FPIC] » : quel standard pour l'accord ? ${ }^{4}$}

10 Parmi les ONG déjà mentionnées pour les rôles qu'elles jouent dans la critique du gouvernement par standard de certification, Forest Peoples Programme a défendu de longue date la notion de "Free Prior Informed Consent" (FPIC dans la suite; en français CLIP : Consentement Libre Informé et Préalable), comme en témoigne son responsable Marcus Colchester (voir son entretien dans ce volume). Ce format d'expression du consentement est aujourd'hui notamment déployé dans le domaine de la santé où l'on requiert en diverses occasions un tel "consentement éclairé » du patient. Dans cette formule et sa pragmatique du choix individuel entre des options publiques, on reconnaît la grammaire libérale composant un public à partir de personnes engagées dans le format d'individus choisissant entre des plans optionnels identifiables par tous. Une telle grammaire ne correspond pas à celles qui ont été traditionnellement développées dans les communautés autochtones, notamment celles affectées par les plantations industrielles de palmiers à huile. Dès notre première rencontre avec Marcus Colchester, nous lui avions soumis ce problème. Il avait répondu que ce cadre normatif ne couvrait pas seulement le mode de consentement d'individus mais aussi celui de peuples, précisant que la notion s'étendait aux droits des peuples à l'auto-détermination.

11 Ce point mérite examen étant donné le poids de la matrice libérale dans le gouvernement par standards, mais aussi dans le droit international et les ONG que nous avons vues à l'œuvre. Par sa formation et son expérience, Colchester est une des personnes les mieux qualifiées pour s'exprimer sur un tel sujet. Après une solide formation en anthropologie (Ph.D. en anthropologie sociale de l'Université d'Oxford, 1982) impliquant un terrain auprès des indiens Sanemas du Venezuela (liés aux 
Yanomamis), puis divers autres missions sur l'impact de projets de développement, il se tourne vers l'anthropologie appliquée ${ }^{5}$. En 1990, il crée puis dirige Forest Peoples Programme (FPP), organisation destinée initialement à lier aux " peuples de la forêt " les ONG concernées par la déforestation. FPP est devenue une organisation reconnue de défense des droits de l'homme et de soutien à l'auto-détermination [self-determination] des "peuples des forêts", appellation désignant à la fois des peuples autochtones et d'autres groupes qui entretiennent des liens de long terme avec la forêt. Colchester connaît parfaitement la tension qui traverse l'anthropologie - comme les sciences humaines plus généralement - entre des approches individualistes et d'autres qui font place à la conscience collective, aux formes sociales et à la communauté imaginée. Son orientation, favorable aux secondes, critique les premières et s'oppose même à une idéologie de "liberté " plus libertarienne et anarchiste que libérale, qui lui semble déformer les travaux d'anthropologues français ayant aussi écrit sur les Yanomamis, Pierre Clastres et Jacques Lizot (Colchester 1982). Il est en outre professionnellement en première ligne pour connaître la tension similaire qui traverse le droit, particulièrement international, dont le pôle individualiste est demeuré longtemps prépondérant. Alors que la Déclaration des droits de l'homme des Nations Unies et les deux Pactes de l'ONU sur les droits civils, politiques, économiques, sociaux et culturels, mettent en avant les droits de l'individu vis-à-vis de l'État, la Charte de l'ONU est fondée sur une notion collective de "droit des peuples» («We, the peoples of the United Nations...»)(Colchester 2002). Si "les outils des droits de l'homme internationaux penchent de manière éhontée en faveur de droits individuels » (« unashamedly individualistic in their emphasis »), l'Organisation Internationale du Travail a établi dès 1957 sa Convention 107 « relative aux populations aborigènes et tribales » qui concerne la protection et l'intégration des populations aborigènes et autres populations tribales et semi-tribales dans les pays indépendants (id.).

12 Dans son combat en faveur de droit des peuples, Colchester doit aussi faire face aux opposants inattendus que sont des collègues anthropologues. Dans une conférencedébat à la London School of Economics, sa contribution intitulée « Affronter Kuper » (« Facing Kuper: Indigenous Peoples, Human Rights and Democratic Development») est prononcée en présence du fameux anthropologue Adam Kuper connu pour sa charge contre l'anthropologie culturelle nord-américaine (Kuper 1999). Colchester doit répondre à l'opposition que ce dernier adresse à l'encontre du concept de « indigenous peoples » pour la raison qu'il supposerait des «peuples primitifs » privilégiés par des "droits spéciaux », et menacerait de les figer dans des mœurs traditionnelles selon une conception « mauvaise » et même " raciste » de l'anthropologie, similaire à la référence par les nationaux-socialistes au sang et au sol (Colchester 2006).

13 En début de son entretien (voir sa publication dans ce volume), Colchester revient sur "les deux formes de consentement qui ont atteint le monde des droits de l'homme ». L'une est un droit individuel établi en réaction aux expérimentations nazies sur les prisonniers. L'autre est un droit des peuples, d'origine différente puisqu'il remonte à la révolution française et à la déclaration américaine d'indépendance, qui ont mis en avant le consentement des gouvernés. Colchester met en relation cette seconde généalogie de FPIC utilisée lorsque les américains se sont engagés dans la deuxième guerre mondiale et le langage de Lénine sur l'auto-détermination, le droit des peuples à l'auto-détermination ayant été repris dans la Charte de l'ONU après la guerre (Colchester, entretien dans ce volume). 
Dans son ouvrage Nazi Medicine and the Nuremberg Trials: From Medical Warcrimes to Informed Consent, Paul Weindling a retracé la première généalogie de FPIC en commençant par les révoltes de prisonniers des camps nazis. Soumis à expérimentation, ils s'étaient ironiquement désignés comme « Lapins » (dans le sens de cobayes) en s'insurgeant contre les expériences qui n'avaient pas de rapport avec leur statut de prisonniers, et en réunissant des témoignages et preuves utilisés ultérieurement dans le procès de Nuremberg (Weindling 2004, 11-26, chap. 1 "The Rabbits Protest»). Dans leur ouvrage sur «Informed Consent", Ruth Faden et Tom Beauchamp suivent le déplacement de cette catégorie qui, issue du monde de la médecine, a fait son apparition dans la jurisprudence nord-américaine en 1957 avant de se répandre plus largement. La première partie de l'ouvrage, consacrée aux fondements de la catégorie FPIC dans la théorie morale, met en avant le principe tout libéral d'autonomie individuelle, celui de justice étant également mentionné quoique demeurant en retrait par rapport à cette autonomie individuelle (id., 7,15). Plus récemment, Pamela Lomelino a revisité les fondements philosophiques de la catégorie FPIC, dans ses relations avec l'autonomie mais aussi avec la communauté (Lomelino 2015). Dans la littérature anglo-saxonne, la critique féministe est la principale et prédominante ressource pour faire ressortir les limites de la conception libérale classique de l'autonomie, et reconnaître des relations de dépendance ${ }^{6}$. A partir des critiques féministes adressées à l'autonomie, Lomelino fait ressortir le manque de reconnaissance du rôle de la communauté et des contraintes qu'elle fait peser sur l'autonomie. Elle considère que les conceptions classiques de l'autonomie qui supportent la catégorie FPIC (1) négligent les relations de dépendance et (2) ne rendent pas compte de l'impact des structures sociales sur l'autonomie (id., 54). Elle développe alors une conception «relationnelle» de l'autonomie en accord avec l'approche de Gerarld Dworkin. Selon sa conception étendue, ce dernier considère qu'une personne ayant comme règle de conduite de suivre les conseils de sa mère, ses amis, son chef ou son directeur de conscience, est autonome (Dworkin 1988, 21). Avec une telle définition normative, les distinctions entre grammaires du commun sont brouillées et donc aussi - ce qui nous importe ici au premier chef - l'analyse des tensions et oppressions qu'un format d'engagement fait peser sur d'autres. Dans une perspective de comparaisons interculturelles, une variante de la catégorie FPIC a été proposée pour la rendre plus ajustée à l'Extrême Orient, changer son orientation de «individual-directed » en "familyoriented " et lui permettre d'embrasser une doctrine confucéenne du soin prodigué aux proches (Fan 2015, 7,11). Cette variante de la catégorie FPIC destinée à la rendre culturellement plus pertinente pour l'Orient, pas plus que l'extension proposée par Gerald Dworkin, n'aident à repérer les tensions entre des constructions pragmatiques profondément dissemblables du différend et de l'accord.

15 Ces remarques nous ramènent à la question posée initialement à Colchester, sur la relation entre la catégorie FPIC et les fondements normatifs de la tradition politique et juridique libérale. Sa réponse, qu'il a développée en début de son entretien (voir dans ce volume), retrace, avons-nous dit, deux généalogies qui seraient couvertes aujourd'hui par la catégorie et "se rencontrent dans le monde des droits de l'homme ", selon son expression. Leurs différences restent cependant considérables et la seconde généalogie des droits des peuples qu'il dessine à partir de la notion de self-determination est elle-même diverse au regard des auteurs qu'il cite. Entre Thomas Paine, dont le cosmopolitisme libéral est déjà une tentative pour conjuguer droit des individus et droits des nations (Lamb 2014), et les positions de Lénine sur le «droit des nations à 
disposer d'elles-mêmes » (Lénine, 1973 [1914]), l'écart est considérable en raison des constructions politiques sous-jacentes. L'emploi d'une commune dénomination FPIC pour couvrir l'ensemble n'est-il pas sans risque?

16 Le choix peut être stratégique pour favoriser l'alliance entre organisations internationales. Mais ne risque-t-il pas d'entretenir un biais en faveur de la compréhension individuelle de l'accord consenti, et de son fondement normatif dans une tradition libérale ? Colchester est conscient de cette difficulté et mentionne à ce propos les tensions fortes entre une grammaire libérale qui sert à promouvoir des droits individuels et des constructions du commun qui font place à des coutumes. Nous $\mathrm{y}$ reviendrons dans la troisième partie où nous considérons la transformation des divers formats évaluatifs et normatifs que réclame RSPO, et la place qu'y tient le cadre FPIC étendu à l'auto-détermination des peuples.

\section{Les fonctions et principes idéaux du gouvernement par standards, comparés à ceux d'un État de droit national}

L'extension mondiale de la culture du palmier à huile selon un modèle industriel, de l'Afrique à l'Indonésie et à la Malaisie qui en produisent aujourd'hui $85 \%$, a suscité des controverses. Les critiques ont porté sur la déforestation, l'extinction de forêts primaires et la menace sur les espèces qui y vivent, la biodiversité, la destruction et la combustion de tourbières qui accroissent le réchauffement climatique, la mainmise de multinationales sur les terres de populations locales et indigènes, le type de modèle agro-industriel déployé à grande échelle qui est porteur d'asymétries et de violence ${ }^{7}$, la violation des droits de l'homme et des droits du travail qui vont jusqu'à des violences physiques. Le standard de certification de l'huile de palme a été créé à l'initiative de WWF et Unilever pour faire face à ces critiques. Evitant les discussions entre experts et entre Etats-nations qu'impliquent les normes ISO, avec le motif d'aller plus vite et d'être plus efficace, le standard cherche à répondre aux critiques portant sur le défaut de légitimité d'instances de normalisation opérant hors de tout débat public. L'ambition de ce nouveau mode de gouvernement par les standards à se substituer aux Etats-nations, sur certaines de leurs prérogatives en matière de défense de droits, invite à comparer ce système normatif à celui d'un État de droit national considéré dans ses fonctions idéales ${ }^{8}$. La prise en compte politique de biens fondamentaux pluriels passe par la fixation d'objectifs et de mesures objectives de leur réalisation, imposant les réductions d'un «gouvernement par l'objectif " (Thévenot 2015b, 2015c). Les lieux d'édiction des normes, les organismes chargés de leur mise en place et de leur contrôle, les principes et critères qui les guident, constituent un nouveau genre de gouvernement privé contrastant avec le gouvernement public d'un Etat assurant des garanties à ses citoyens par des pouvoirs législatif, judiciaire et de police. A la normativité du droit est privilégiée la normalisation d'un produit et de sa production.

\subsection{Textes normatifs}

18 Le cœur du gouvernement par les standards tient à l'exposé du standard lui-même qui fait office de texte normatif principal. L'exposé du standard est organisé hiérarchiquement autour de huit "principes » supérieurs décomposés en "critères ", 
« indicateurs » et " lignes directrices ». Considérons ce texte, en portant attention aux ajouts, lors de la révision du standard en $2013^{9}$, qui témoignent de points ayant suscité des critiques et des modifications acceptées.

\section{Tableau 1 : Textes normatifs}

\begin{tabular}{|l|l|}
\hline $\begin{array}{l}\text { Gouvernement } \\
\text { représentatif }\end{array}$ & $\begin{array}{l}\text { Gouvernement par le standard RSPO et mention de quelques modifications } \\
\text { opérées en } 2013\end{array}$ \\
\hline Constitution & $\begin{array}{l}\text { «Transparency, inclusiveness, consensus » codifiés par ISEAL (International } \\
\text { Social and Environmental Accreditation and Labeling Alliance) }\end{array}$ \\
\hline
\end{tabular}




\begin{tabular}{|c|c|}
\hline $\begin{array}{l}\text { Lois } \\
\text { Textes } \\
\text { d'application }\end{array}$ & 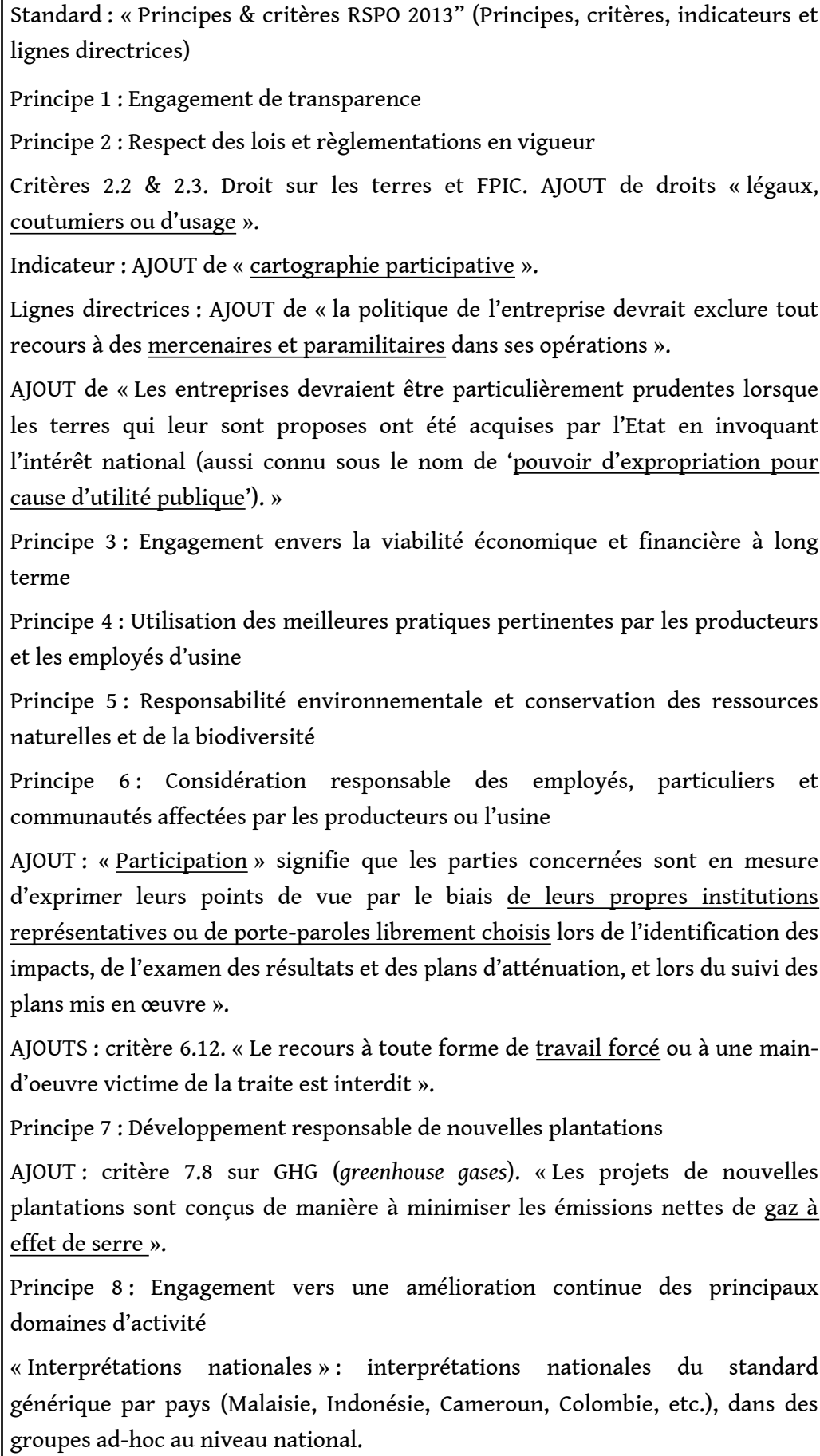 \\
\hline
\end{tabular}

Les huit principes sont hétéroclites au regard de normes juridiques, mais leur ordre indique une certaine priorité. Conformément à sa place, le premier d'entre eux se trouve en position supérieure puisqu'il porte sur les exigences de publicité et de communication dans la procédure (1. Engagement de transparence). Il reprend un semblable principe avancé par l'ISEAL (International Social and Environmental Accreditation and Labeling Alliance): "transparency, inclusiveness, consensus». En revanche, le deuxième ne manque pas de surprendre le juriste ou même le citoyen ordinaire puisqu'il stipule que les stakeholders s'impliquant volontairement dans le 
standard s'engagent à... respecter la loi (2. Respect des lois et réglementations en vigueur). Les ajouts en 2013 aux critères relevant de ce deuxième principe révèlent des pratiques contrevenant à la loi. Dans les «lignes directrices» (pour mesurer un indicateur), est ajoutée la prohibition de l'usage par les compagnies de mercenaires et de para-militaires qui font violence aux paysans : «La politique de l'entreprise devrait exclure tout recours à des mercenaires et paramilitaires dans ses opérations ». Dans les indicateurs, un ajout fait référence à une exigence ne figurant pas dans les droits nationaux mais dans des conventions internationales : la " cartographie participative", qui suppose de cartographier les droits d'usage et coutumiers existant sur les terres avec les communautés ("parties affectées »). Un autre ajout mentionne expressément que des offres par l'État peuvent ne pas satisfaire des conventions internationales voire le droit national: «Les entreprises devraient être particulièrement prudentes lorsque les terres qui leur sont proposées ont été acquises par l'Etat en invoquant l'intérêt national (aussi connu sous le nom de "pouvoir d'expropriation pour cause d'utilité publique ») $»^{10}$. Les critères $2.2 \& 2.3$ concernant le "Droit sur les terres » et le Free Prior Informed Consent ont été complétés par la considération plus variée des droits "légaux, coutumiers et d'usage ", ouvrant à une prise en compte plus claire des droits coutumiers relatifs à l'usage de la terre ${ }^{11}$.

Les deux principes suivants reviennent quant à eux au cœur originel du standard, tel qu'il fut initialement conçu par les compagnies impliquées, et orienté par les grandeurs marchande et industrielle. Le principe 3 porte sur «l'engagement envers la viabilité économique et financière à long terme ». Le principe 4 précise, dans un grand détail, les "meilleures pratiques pertinentes" de production et de transformation (gestion de l'eau, des pesticides, des sols, du risque environnemental et sanitaire, etc.) sans remettre en cause le modèle d'agriculture industrielle, jugé plus productif (principe 3). Cette normativité descend bien en-dessous du niveau des justifications publiques (selon des grandeurs justifiées par un bien commun) et emprunte le format d'évaluation du plan en prenant appui sur des méthodes et équipements qualifiés fonctionnellement pour soutenir ces plans. Cette minutieuse prescription de tâches techniques inscrites dans le standard n'a pas son équivalent dans le droit.

Le principe 5 introduit une toute autre grandeur, verte (Blok 2013, Lafaye \& Thévenot 1993), ainsi que les valeurs connexes défendues initialement par l'ONG environnementale WWF instigatrice du standard avec Unilever: "Responsabilité environnementale et conservation des ressources naturelles et de la biodiversité ».

C'est avec le principe 6 que viennent en considération les biens et maux des acteurs humains les plus vulnérables de la filière, les paysans et leurs communautés ainsi que les employés des plantations et des entreprises de transformation: "Considération responsable des employés, particuliers et communautés affectées par les producteurs ou l'usine ». Notons qu'à l'obligation de respecter des droits sociaux est substituée la " considération responsable». Les ajouts au standard en 2013 indiquent quelques points sensibles. Notamment, le travail clandestin et forcé déjà mentionné comme interdit dans la version de 2007 - quoique seulement en tant que «ligne directrice »apparaît désormais comme "critère" 6.12: «Le recours à toute forme de travail forcé ou à une main-d'œuvre victime de la traite est interdit».

Le principe (6) est d'abord envisagé (6.1) à partir de l'outil standard des «études d'impact social» (indicateur 6.1.1) et des procédures pour atténuer («mitigate») ces impacts, qui empruntent le format de "plans $»^{12}$. C'est seulement dans la rubrique 
«lignes directrices » que l'on repère les marques du combat mené par l'ONG Forest Peoples Programme, aux côtés d'autres ONG telles qu'OXFAM Novib, pour que soient stipulées des, modalités d'accord reposant sur le concept de Free Prior and Informed Consent: « Participation signifie que les parties concernées sont en mesure d'exprimer leurs points de vue par le biais de leurs propres institutions représentatives ou de porte-paroles librement choisis lors de l'identification des impacts, de l'examen des résultats et des plans d'atténuation, et lors du suivi des plans mis en œuvre ». On remarque la considération dans les «impacts sociaux potentiels" de "valeurs culturelles et religieuses » et « autres valeurs communautaires ». Le critère suivant 6.2 porte entièrement sur l'accord en promouvant des « méthodes de communication et de concertation ouvertes et transparentes ». Les indicateurs se concentrent sur l'enregistrement écrit des consultations et communications (6.2.1), la nomination d'une personne responsable chargée de ces questions (6.2.2) et l'établissement d'une «liste des parties prenantes» (6.2.3). Remarquons que dans les lignes directrices, le développement des exigences de consultation et communication, avec les communautés locales notamment, incite à emprunter la forme du «forum multiparties prenantes ", qui peut s'avérer incompatible avec les modalités traditionnelles d'expressions de l'accord. Les critères suivants portent sur l'existence d'un mécanisme de «traitement des plaintes et des revendications» (6.3), puis sur le point majeur des « négociations relatives à l'indemnisation pour la perte de droits légaux, coutumiers ou d'exploitation [...] au moyen d'un système documenté qui permet aux peuples autochtones, aux communautés locales et aux autres parties prenantes d'exprimer leur avis par le biais de leurs propres institutions représentatives » (6.4).

Avec le critère 6.5 sont introduites la rémunération minimale - pour ne mentionner que la loi ou les conventions collectives («au moins les normes minimales légales ou industrielles »), les conditions de travail et de vie, et notamment l'épineuse question de l'approvisionnement pour la vie quotidienne : « Les producteurs et les employés d'usine doivent déployer des efforts tangibles pour contrôler et améliorer l'accès des travailleurs à une alimentation adéquate, suffisante et abordable »(6.5.4). Dans le cas courant où les travailleurs logés par la compagnie sur la plantation sont obligés de faire appel à la boutique de la dite compagnie, faute de possibilités de se déplacer vers d'autres commerces, on sait que les prix élevés pratiqués permettent à celle-ci de récupérer une part significative du salaire versé. Un rapport d'audit de 2012 rappelle celui de 2009 qui signalait déjà pour la même compagnie que "no formal action has been taken to address the issue of high grocery prices [and] the prices of food at the [oil mill] cafeteria ", mentionnant que "In the interest of the workers and also staffs, it is felt that some concerted efforts be undertaken to address the issues raised". Au sujet des prix eux-mêmes, le rapport d'audit ne peut que signaler des absences d'étiquettes dans le magasin, se concentrant comme le plus souvent(Power 1997) sur les procédures d'enregistrement et d'inscription écrite (« records ») $)^{13}$.

Les critères suivants précisent des droits relatifs aux quatre catégories de droits fondamentaux au travail de l'OIT ${ }^{14}$, avec la liberté syndicale et le droit de négociations collectives (6.6.), l'interdiction du travail des enfants (6.7.) et du travail forcé (6.12) et l'élimination des discriminations en matière d'emploi (6.8.), en ne se référant toutefois explicitement (dans les lignes directrices) qu'à trois des huit conventions fondamentales de ces droits ${ }^{15}$. Enfin, 2 critères portent sur l'élimination du harcèlement (notamment des femmes) et l'engagement à «respecter les droits de 
l'homme» (6.13), dont l'indicateur unique (6.13.1) tient à ce qu'une "politique interne » soit « documentée et communiquée » au personnel.

En somme, après un principe 1 procédural et un principe 2 engageant au respect du droit national et d'éléments de droit international, les principes 3, 4, 5 et 6 tendent à intégrer un pluralisme de biens dans le standard et donc dans les propriétés supposées mesurables du produit et de sa filière de production ${ }^{16}$.

Au fil des négociations avec les ONG, ces principes se sont progressivement ouverts à la prise en compte d'une variété de droits (y compris de droits coutumiers). Mais leur application concrète reste questionnée. En matière de travail par exemple, sous l'influence des ONG sociales, le standard intégrait dès sa première version en 2007 des principes et droits fondamentaux (avec notamment la mention, dans les critères, de la liberté syndicale, de la non-discrimination et de l'interdiction du travail des enfants). Au moment de la conférence annuelle de 2013, une coalition d'ONG et de syndicats de travailleurs a pourtant manifesté dans les rues pour faire état de violations de droits des travailleurs dans des plantations, y compris dans des plantations certifiées. Le même jour, dans la salle de conférence, plusieurs groupes de participants se sont réunis pour mettre à l'ordre du jour le droit des enfants, le droit des femmes - notamment contre le harcèlement - et plus largement le droit au travail. Ces réunions prolongeaient les efforts du groupe de travail RSPO «Human right », animé par Oxfam et une multinationale, et parallèlement, de « l'alliance des travailleurs ». Les protestations et nouvelles mesures prises témoignent d'une critique de l'absence d'effet de ces mentions antérieures du droit du travail dans la norme. D'une manière générale, elles posent la question épineuse de la mise en œuvre du standard (voir aussi l'entretien de Colchester) et des pouvoirs et fonctions légitimes.

\subsection{Souveraineté : pouvoirs et fonctions légitimes}

Le second tableau comparatif traite de la légitimité des pouvoirs établis par chacun des deux systèmes normatifs que nous mettons en parallèle (tableau 2). Le standard RSPO vise une légitimité dépassant celle d'accords contractuels bilatéraux. Il peut se targuer de reposer sur une large base [constituency] qui réunit $40 \%$ des acteurs de la production mondiale de l'huile de palme. Afin d'assurer cette légitimité, le standard déploie un dispositif «multi-stakeholder» supposé donner une voix égale à des individus porteurs des intérêts multiples en jeu, répartis en l'occurrence en sept catégories : planteurs, industriels et négociants de l'huile, producteurs de biens de consommation, commerces et notamment chaînes d'hypermarché, banques, ONG environnementales, ONG sociales.

L'exécutif («Board») joue un rôle primordial dans la fixation de l'ordre du jour des conférences et dans l'organisation générale, et reflète l'exigence de participation des intérêts à la recherche d'un équilibre entre les 7 catégories de stakeholders. En dehors du Board, plusieurs centaines de participants sont appelés à s'exprimer, lors des conférences annuelles, dans des groupes de travail, et en Assemblée Générale. Les conférences annuelles proposent un format très classique de séances plénières comportant des exposés de la part de quelques membres choisis, mais aussi des formes d'échanges issues du management participatif tels que "open space technologies » et «world cafés». La grammaire politique libérale souvent dite "horizontale» (tous les porteurs d'intérêt sont traités sur le même plan) rencontre de fait d'énormes asymétries, entre multinationales et petits paysans de Sumatra ou Bornéo par exemple. 
La critique courante et pertinente porte sur les rapports de force qui en résultent et qui vont à l'encontre de l'horizontalité affichée. Les possibilités d'échange et de construction d'un accord offertes par ce dispositif, qui amènent les petits planteurs à s'y engager cependant, génèrent aussi des oppressions moins visibles et plus insidieuses sur lesquelles ont porté nos investigations.

Tableau 2 : Souveraineté, pouvoirs et fonctions légitimes

\begin{tabular}{|c|c|}
\hline $\begin{array}{l}\text { Gouvernement } \\
\text { représentatif }\end{array}$ & Gouvernement RSPO \\
\hline $\begin{array}{l}\text { Communauté de base des } \\
\text { citoyens (constituency) }\end{array}$ & $\begin{array}{l}\text { Participants à la roundtable: } 40 \% \text { de la production mondiale de l'huile } \\
\text { de palme }\end{array}$ \\
\hline $\begin{array}{l}\text { Expression des voix par } \\
\text { représentants élus }\end{array}$ & $\begin{array}{l}7 \text { catégories de stakeholders ayant une égale voix individuelle: } \\
\text { 1. Oil Palm Growers, } \\
\text { 2. Palm Oil Processors and/or Traders, } \\
\text { 3. Consumer Goods Manufacturers, } \\
\text { 4. Retailers, } \\
\text { 5. Banks and Investors, } \\
\text { 6. Environmental/Nature Conservation NGOs, } \\
\text { 7. Social/Developmental NGOs } \\
\text { Ni représentants des Etats-nations, ni de la recherche. }\end{array}$ \\
\hline Exécutif & $\begin{array}{l}\text { «Executive Board » composé de } 16 \text { sièges représentant les } 7 \text { catégories } \\
\text { ci-dessus, et renouvelable (ont notamment été membres, au cours de } \\
\text { plusieurs mandats: WWF, Unilever, Oxfam). }\end{array}$ \\
\hline $\begin{array}{l}\text { Délibération par débat } \\
\text { public }\end{array}$ & $\begin{array}{l}\text { Importation de méthodes de management: } \\
\text { stands, «world cafés", "open-space », et «working group » animés dans } \\
\text { une logique de projet. }\end{array}$ \\
\hline Police & Audit \\
\hline $\begin{array}{l}\text { Justice, règlement de } \\
\text { conflits }\end{array}$ & $\begin{array}{l}\text { Dispute Settlement Facility, Complaint System } \\
\text { La sanction la plus haute est le retrait de la certification }\end{array}$ \\
\hline
\end{tabular}

Les échanges favorisés par les "open space technologies" incitent les participants volontaires à se saisir d'une pancarte pour y inscrire une question et agréger autour d'eux d'autres participants intéressés par le thème. Sur ce "marché » des pancartes offrant autant de groupes de discussion, voisinent des professionnels d'ONG rompus aux techniques de la participation et des personnes qui n'y sont pas du tout habituées. Certains groupes de discussion peuvent être l'occasion de témoignages rares. Ainsi avons-nous observé des auditeurs procédant à l'autocritique de leurs pratiques en raison de leur dépendance aux firmes auditées qui les rémunèrent et tendent à guider leur travail. Les représentants de "petits producteurs " indonésiens se montrent en revanche embarrassés pour s'engager dans ce dispositif concurrentiel. Le "world café » est une autre technique proposée dans laquelle les participants sont réunis pendant 20 
minutes autour d'une table, à la manière d'un "speed-dating ", pour faire entendre leurs opinions et préférences sur une question, avant de changer de table et de question. Ces formats, bien ajustés à un marché d'idées et à une connexion éphémère dans l'échange, réduisent cependant la teneur des préoccupations apportées par les personnes affectées (Cheyns 2011, Thévenot 2019).

Mais quels organes font fonction de police et de justice? Sur ces fonctions, les différences sont considérables avec un État de droit. Ce gouvernement par les standards ne dispose en effet pas d'organe d'exercice légitime de la force, alors même que ne sont pas rares les violences exercées par des mercenaires au service des compagnies. Les seuls éléments de police empruntent la modalité managériale de l'audit, qui emprunte le format normatif d'objectifs se prêtant à une vérification objective, comme nous l'avons vu dans le tableau 1. L'indépendance douteuse de l'audit est une importante limite dans la réalisation de cette fonction de police, puisque son financement onéreux est assuré par les entreprises susceptibles d'être pénalisées et non par un fonds indépendant (Silva-Castenada 2015). Au-delà du conflit d'intérêts potentiel, les formats de preuve reconnues et valorisées par les auditeurs, parce que centrées sur la réalisation de plans, laissent peu de place à l'expression des maux et des formats de preuves formulés localement par les communautés affectées (Idem). L'audit relève des écarts et des infractions à la norme selon la modalité du " tick the box ", de la case de l'objectif cochée ou non, format digital par excellence préparant à la quantification ${ }^{17}$.

La fonction de justice et de règlement de conflits est aussi toute différente. RSPO a mis en place deux systèmes : le « RSPO Complaints System » de traitement des plaintes, et le « Dispute Settlement Facility » qui repose sur la médiation. Dans aucun des deux ne figurent de juges entendant contradictoirement les parties en conflit et les experts évaluant les cas sont des stakeholders. Ce n'est qu'en 2013 qu'ont été décrétées incompatibles l'appartenance au Complaint panel (instance d'adjudication) et l'appartenance au Board (exécutif). Nous reviendrons sur les pratiques de traitement des conflits dans la dernière section.

\subsection{La " police » de l'audit: « who watches the watchman? »}

Bien que réalisé par une tierce partie, l'audit est accompagné de mécanismes de contrôle des auditeurs, censés faire face à ses défaillances. Selon des règles institutionnalisées, les auditeurs RSPO sont accrédités et contrôlés par une instance supérieure, l'accréditeur (lui-même reconnu et validé par l'instance RSPO). Des défaillances, y compris dans ce système de contrôle, conduisent de nombreuses ONG à contrôler elles-mêmes l'audit. Des ONG extérieures, des journalistes ou représentants de la société civile, exercent ainsi une pression de contrôle, de façon non explicitement attendue par les opérateurs de la RSPO. Cette division du travail peut faire l'objet d'une stratégie explicite entre ONG membres et non membres de la table ronde, au sein de coalitions d' $\mathrm{ONG}^{18}$.

Dès le lancement de la RSPO, Greenpeace a exercé une pression de l'extérieur, en constituant une menace sérieuse pour les entreprises, en particulier pour les actionnaires et investisseurs. Lancée en 2009, la campagne «Burning up Borneo » est emblématique de la menace médiatique que représente Greenpeace pour les acheteurs européens. Dans une stratégie de «conflit-coopération» (Valor et Merino de Diego, 
2009), cette menace a eu pour effet d'inciter les entreprises de la filière à collaborer avec d'autres ONG plus " coopératives » (ou " collaboratives »), telles que WWF, leader des tables rondes, et à rejoindre ces dernières (RSPO, RTRS, etc.) : « Quand une entreprise est réticente, on peut dire: regardez à l'extérieur [de la table ronde] ce qui vous attend [en parlant de Greenpeace] !» (Entretien, WWF Pays-Bas 2009).

Cette stratégie, dite "du bon et du méchant policier», a été documentée et analysée comme un préalable à la mise en œuvre des «soft régulations» que sont les standards privés (Idem). Dans le système de surveillance mondiale par audit, le rôle des ONG extérieures à la RSPO est apparu dès la mise en marché de certificats de plantations d'huile de palme durables RSPO. En 2008, Greenpeace a produit un rapport ${ }^{19}$ dénonçant la première entreprise certifiée RSPO, pour violation du standard RSPO de protection environnementale et sociale dans certaines de ses plantations. Elle y a relevé, à partir de ses propres investigations de terrain, que l'entreprise ne respectait pas certains des critères RSPO requis dans le cadre de la certification partielle de ses plantations. Dans ce rapport, Greenpeace promeut la mise en place de moratoires sur les forêts, mais ne remet toutefois pas en cause le dispositif RSPO, que d'autres ONG, dans une posture collaborative, développent (WWF notamment).

38 L'efficacité du système de surveillance mondiale mis en œuvre par la RSPO a été régulièrement contestée par la suite, notamment dans les années 2013-2016, par des ONG extérieures, non sans lien avec des ONG membres de RSPO. Bien que n'ayant pas participé à l'élaboration de la norme RSPO, ces ONG, à l'aide de leurs rapports, montrent qu'elles en contrôlent son application en contrôlant les certificateurs, c'est-àdire « en contrôlant les contrôleurs ».

En 2013, le rapport «Conflict or Consent? $»^{20}$ a été produit par 19 ONG, des plus locales aux plus internationales (dont Forest Peoples Programme), membres et non membres de RSPO. Il a rendu ainsi visibles une variété de maux et violations de droits dont souffrent des communautés indonésiennes, mettant en accusation des entreprises certifiées $\mathrm{RSPO}^{21}$. Le non respect du FPIC par des sociétés de plantations certifiées RSPO était particulièrement visé. Les ONG signataires ont rendu public ce rapport (conférences de presse) juste avant la conférence annuelle RSPO et l'ont discuté, avec d'autres ONG, dans un atelier préalable à la conférence, afin de définir un plan d'action commun. C'est aussi cette même année qu'une coalition d'ONG et de syndicats de travailleurs locaux, nationaux et internationaux, a organisé une manifestation de rue aux portes de la conférence RSPO, à Medan, pour faire état du non respect des droits fondamentaux du travail dans les plantations de palmiers à huile, malgré l'intégration de ces droits dans la norme depuis 2007.

En novembre 2015, les ONG Grassroots et EIA produisent un rapport critiquant le travail des auditeurs ainsi que leurs capacités à identifier et rendre visibles des dommages et violations des droits des communautés ("Who Watch the Watchmen? »2). Tout comme le précédent rapport mentionné («Conflict or consent?»), celui-ci est stratégiquement diffusé à la presse quelques jours avant le lancement de la Xième conférence RSPO à Kuala Lumpur. Cette même année, un journaliste du Wall Street Journal publie une enquête faisant état de travail forcé dans des plantations auditées et certifiées RSPO en Malaisie, que détient la puissante organisation gouvernementale FELDA ${ }^{23}$ (voir infra). Un an plus tard, en Novembre 2016, Amnesty International révèle des abus violant les droits fondamentaux du travail (notamment par travail forcé), dans d'autres plantations certifiées RSPO, ici détenues par Wilmar, un conglomérat Malaisien. 
41 Par cette contestation de l'efficacité de l'audit à assurer des biens fondamentaux les ONG ont produit plusieurs effets. En 2013, elles ont obtenu du Board de la RSPO une révision du mécanisme de traitement des plaintes (y compris de son format) - voir infra section 3.3 «La transformation des formats ». Elles ont aussi conduit à faire voter en Assemblée Générale une résolution concernant l'indépendance des membres statuant sur les plaintes (résolution $6 \mathrm{f}$ en 2013). Cette résolution a permis pour la première fois de sanctionner, au niveau le plus élevé, des entreprises membres en suspendant ou retirant leurs certifications - sanctions les plus élevées dans le système normatif RSPO.

Ainsi, l'enquête du journaliste du Wall Street Journal en novembre 2015, révélant des cas de travail forcé dans des plantations certifiées RSPO, a conduit, après une série d'actions d'ONG et du Complaint Panel de RSPO, au retrait de certificat du plus grand producteur mondial de palmiers à huile $(810000 \mathrm{ha})$, membre influent de la table ronde (FELDA, membre du Board depuis 2006). En effet, cette enquête fut immédiatement relayée par une coalition d'ONG internationales dans un communiqué de presse, appelant la RSPO à engager une "vérification indépendante ", et par le Complaint Panel de RSPO qui a déposé plainte contre les 3 plantations de FELDA mises en cause. Cette plainte a eu pour effet d'ordonner une procédure de vérification par ASI, l'organisme accréditeur qui avait accrédité le certificateur mis en cause. Dans un premier temps, le rapport d'ASI n'a pas rapporté de défaillance en ce qui concerne l'accusation de travail forcé sur les plantations, même s'il mentionnait d'autres défaillances de l'audit ("major non compliance »). Mais il a été publiquement critiqué par des ONG extérieures, pour n'avoir identifié aucune « forme d'évidence de travail forcé » alors qu'il mentionnait de nombreux éléments internationalement reconnus comme indicateurs de travail forcé (notamment les papiers d'identité des travailleurs étaient détenus par le management de la société de plantation). Au vu d'une série de rapports de vérifications, le complaint panel a pris la décision de suspendre le certificat d'une des 3 plantations (le temps pour elle de se conformer). Deux mois plus tard, c'est finalement l'entreprise elle-même qui a décidé de renoncer au certificat de l'ensemble de ses 58 plantations, tout en s'engageant dans un plan d'action destiné à rétablir sa conformité au cahier des charges (qui fera l'objet d'une nouvelle vérification).

43 L'année suivante, en 2016 , c'est le groupe IOI $^{24}$, autre membre fondateur de RSPO, qui a vu son certificat suspendu durant 3 mois, à partir d'une plainte déposée en 2015 par une ONG environnementale (AIDEnvironement), quant au non respect de critères environnementaux (cas Ketapang). IOI a toutefois récupéré son certificat 3 mois après la suspension, une fois son plan d'action rectificatif, prévu sur 1 an, approuvé par le Complaint Panel ${ }^{25}$. Ce cas signale aussi l'écart entre la construction multi stakeholder de «voix égales» et l'existence d'asymétries de pouvoir, que les ONG tentent de contrecarrer non sans déployer d'importantes ressources. En effet, le cas Ketapang avait déjà fait l'objet d'une plainte par la même ONG en 2010, qui s'était réglée par un avis de bonne conduite d'IOI émis par le Board, alors encore souverain :

"With regards to Ketapang, the RSPO Grievance Panel has concluded that there is insufficient evidence to prove that HCV areas were deliberately cleared by IOI in Ketapang or that the estates involved were knowingly proceeding to clear land without being in possession of all permits required (lettre signée par le secrétariat exécutif suite à décision du Board).

En réaction, AIDEnvironnement avait contesté une manœuvre rhétorique du Board consistant à « inventer un langage ${ }^{26}$ pour protéger IOI (la qualification d'un acte 
commis "délibérément et en connaissance de cause ») et signalé une incapacité du Board à sanctionner ses membres, notamment ceux siégeant en son sein (IOI) :

«In an apparent attempt to justify its unwillingness or inability to enforce RSPO's written rules on IOI Group, RSPO's Grievance Panel frivolously invented language that does not exist in RSPO's standards and procedures ('deliberatel' and 'knowingly') ».

Jusqu'en 2013, les propositions de sanctions du panel devaient en effet être approuvées par le Board. C'est ce que la résolution 6f, votée en 2013 et proposée par une coalition d'ONG, est venue modifier, en réclamant que les membres qui statuent sur les plaintes soient indépendants de l'organe de décision que constitue le Board (conseil d'administration). Le vote de la résolution of en 2013 a nécessité une grande préparation et coordination d'ONG, qui ont dû au préalable constituer des documents rapportant des « réalités du terrain ", se coordonner dans le cadre d'ateliers de travail, mener une campagne et lancer une pétition. Suite à l'adoption de la résolution 6f, AIDEnvironnement a pu redéposer une plainte contre IOI sur le cas Ketapang, qui a cette fois aboutit à une sanction.

Dans le rapport de force stratégique, les ONG essayent en outre de peser sur les tentatives de désinvestissement qui restent, pour certaines entreprises, un recours possible afin d'échapper aux sanctions ou se libérer d'engagements antérieurs issus de négociations. Le cas mentionné dans l'entretien de Marcus Colchester (voir dans ce même volume), qui n'est pas un cas isolé, concerne une autre plainte déposée contre IOI (IOI-Pelita). Celle-ci, déposée en 2010 par les communautés de Long Terang Kanan et l'ONG Grassroots, expose un conflit foncier qui dure depuis plus de 20 ans, et vise des abus des droits des communautés locales (droits de l'homme, FPIC). La plainte avait donné lieu à nombreuses procédures de résolution, y compris la médiation que propose le « Dispute Settlement Facility » de RSPO. Des ONG internationales ont condamné en 2017 le projet de désinvestissement de IOI Pelita, qui intervenait après 6 mois de négociations et de finalisation d'un plan d'action approuvé par les parties :

"If IOI sells its stake in IOI-Pelita it would be the final straw, proving once and for all that IOI cannot be trusted. If this sale goes through, then major brands would have no choice but to stop buying from IOI altogether. The RSPO would also have to expel IOI for such a gross violation of its rules," said Bagus Kusuma, forest campaigner for Greenpeace Southeast Asia." ${ }^{27}$

\section{L'accord, les plaintes et les maux transformés dans les formats du standard}

Nous avons exposé les limites importantes de ce gouvernement qui tiennent à sa faible capacité d'imposer ("enforcement») les dispositions du standard. L'engagement est volontaire et, avons-nous souligné, les fonctions de police et de justice se trouvent réduites dans ce gouvernement non étatique. Dans cette dernière partie, nous allons pousser plus loin l'analyse des limites en portant attention aux exigences de transformation des préoccupations des communautés locales, que requièrent le standard RSPO et son gouvernement pour que ces préoccupations soient recevables, qu'elles portent sur l'accord ou la prise en compte de maux dans le traitement du différend. Notre cadre d'analyse des divers « formats » de biens et de preuves engagés permet de décomposer la chaîne des transformations requises et les sacrifices qu'elles imposent. 
51 Nous avons souligné que le standard RSPO prend appui sur le format du plan, tout en s'ouvrant progressivement à la considération de droits, et en particulier de droits collectifs et coutumiers. Quelles tensions naissent de la rencontre de ces différents formats de normativité engagés? Comment les atteintes et maux affectant les populations les plus vulnérables sont-ils pris en compte dans ce gouvernement RSPO ? La réponse à ces questions passe par la mise au jour de divers mécanismes conduisant à des décalages, distorsions voire empêchements dans la chaîne des transformations que suppose cette prise en compte.

\subsection{Tensions avec les formats de droits}

52 Commençons par la transformation des préoccupations dans le format de droits, qui dépasse le cadre de RSPO et que l'on retrouve dans les diverses contributions rassemblées en ce recueil. Qu'impose de mettre en forme de droits ce qui soucie les personnes - parmi les plus vulnérables - couramment engagées pour des biens dans un tout autre format? Le socle libéral du droit moderne, et notamment des droits de l'homme devenus la lingua franca des Etats aussi bien que des ONG repose sur l'engagement volontaire d'un sujet autonome. Ils relèvent en cela de la grammaire libérale régissant une communauté d'individus choisissant, qui contraste avec d'autres constructions du commun accueillant des engagements autres (Thévenot 2014, 2015a). La figure d'autonomie fait ainsi violence aux dépendances mises en valeur dans l'attachement familier ou spirituel des habitants à des lieux chers tels qu'une forêt dont une communauté a la charge de prendre soin. Le mouvement d'extension de droits individuels à une nouvelle génération de droits collectifs de populations indigènes, depuis les années 1970, vise à remédier à cette limitation. Les conventions internationales de l'après-guerre ont promu une première et deuxième génération de droits de l'homme qui mettaient en avant des droits individuels et renforçaient la propriété privée et le droit positif plutôt que la coutume. A l'instigation de représentants de peuples indigènes, une troisième génération a contribué à l'élargissement à des droits collectifs de peuples et à leur auto-détermination. Marcus Colchester met en avant cette ouverture émancipatrice qu'accompagnent ses efforts, non sans mentionner une histoire longue de la place faite par les empires et les puissances coloniales aux coutumes des peuples sous leur emprise, montrant que la reconnaissance peut aller de pair avec la perpétuation d'une mainmise (Colchester 2011). Il souligne aussi le contexte d'après guerre, puis de guerre froide, qui a favorisé la conception libérale de droits protégeant l'individu d'un Etat oppressant son autonomie individuelle. Karen Engle - qu'il mentionne - a mis en évidence « l'architecture fragile» de la déclaration des Nations Unies sur le droit des peuples indigènes (UNDRIP, UN Declaration on the Rights of Indigenous Peoples) de 2007. Elle précise que ce compromis instable avec le socle libéral conduit à une limitation en même temps qu'à une reconnaissance de formes d'auto-détermination des peuples indigènes, le « néolibéralisme » «multiculturel» s'accommodant très bien de droits culturels indigènes (Engle 2011, p. 160-161). Marcus Colchester, qui a œuvré sans relâche pour l'extension du consentement à celui « des peuples » dans les certifications mais aussi dans les plantations en général, les constructions de barrages et les mines, est conscient des tensions que suscite le format normatif FPIC (Colchester 2002), qui porte l'empreinte de la grammaire libérale, avec la généalogie double que nous avons rappelée. 

formel et de la décision claire dont on attend qu'ils closent une négociation, objectif premier des compagnies dans leurs relations avec les communautés. Les communautés, en revanche, souhaitent établir une relation de confiance avec de nouveaux voisins, qui dépasse la question de l'usage de leurs terres. C'est pourquoi il promeut l'idée d'un processus itératif, qui permet aux parties de faire des concessions mutuelles plutôt que de simplement signer un document de cession des terres (entretien dans ce volume). Ce dernier point nous amène à considérer maintenant les tensions entre les préoccupations indigènes ou locales et le format du plan, plutôt que celui des droits de l'homme.

\subsection{Tensions avec les formats du plan et de l'objectif}

L'objectif d'une décision objectivée demeure la visée des compagnies, avons-nous souligné, selon un format de gouvernement par le plan et sa réduction à l'objectif. or l'ouverture de FPIC à la coutume et à l'auto-détermination suscite des tensions avec ce régime du plan réifié dans l'objectif. Les compagnies demandent de disposer d'une 
procédure d'opération standard, unique et rapide, pour l'application du FPIC. Elles veulent pouvoir « cocher des cases » (« tick the box ») pour objectiver la réalisation d'un plan. A cela Colchester répond que le FPIC, cherchant à impliquer les membres de la communauté concernée, ne peut être simplement inscrit dans un plan standard, et suppose une grande variabilité des façons de s'enquérir du consentement : " There is not AN answer. There have to be different answers for different circumstances and different peoples. (...) You cannot have a standard operating procedure for how you do FPIC, because it is their operating procedure, not yours » (voir son entretien dans ce volume).

Pour faire valoir un FPIC élargi aux peuples, introduisant des principes normatifs substantiels qui sont pour partie étrangers au socle libéral, les ONG font ainsi face à une tentative de recadrage du FPIC dans le format du plan. Colchester évoque dans l'entretien non seulement les difficultés d'interprétation du FPIC par les compagnies, mais aussi leurs interventions qui ont rendu l'élaboration du guide d'application du FPIC extrêmement procédurale. Le format «tick the box» de la checklist est de même ajusté aux compétences des auditeurs tournées vers la quantification et le contrôle de documents formels ou écrits déjà apprêtés pour le format de plans. Le rapport «Who watch the watchmen" (2015) critiquait l'incapacité des auditeurs "à identifier des revendications de droits sur les terres de communautés indigènes et le risque de travail forcé ». De même Colchester souligne que les auditeurs, s'ils se prêtent facilement à vérifier ce qui est dans le format du droit national (tel qu'un permis d'usage légal), l'existence d'un "business plan » ou l'exécution d'une tâche comme le défrichement de la forêt, ont beaucoup plus de mal à savoir si les gens sont satisfaits de leurs conditions de travail, ont été traités équitablement, et si les femmes ont été harcelées en particulier. Il mentionne par ailleurs le recours fréquent aux « groupes de travail » de RSPO, qui reposent sur un travail technique et une « multitude de détails », écartant la participation des ONG locales.

Des ONG peuvent ainsi soumettre le gouvernement RSPO à l'épreuve critique grâce à des modalités d'action variées (lors de conférences de presse, d'ateliers auxquels sont conviés les intéressés, voire de manifestations de rue). Néanmoins ces actions sont ensuite fréquemment recadrées et traitées dans le format du plan, tendant à réduire la portée de leurs critiques ou des principes substantiels mis en avant.

\subsection{La transformation des formats pour faire remonter les préoccupations et plaintes locales}

61 Une longue et complexe chaine d'ONG est requise pour inscrire de nouvelles questions à l'ordre du jour, faire remonter des plaintes locales sur une scène transnationale, ou les instruire vers le mécanisme de traitement des plaintes. Quoique non explicité dans le gouvernement par le standard, la dynamique du gouvernement RSPO repose sur une charpente d'ONG qui n'opèrent pas seulement comme stakeholders ou en défense d'un bien commun environnemental. Alors que des ONG multinationales interagissent de pair à pair avec des entreprises multinationales en privilégiant les mêmes formats d'évaluation les plus formalisés et les plus publics, d'autres se montrent capables d'épouser au plus près le format d'attachement le plus local et particularisé grâce à leur liens avec des acteurs de terrain et à leur implantation locale. Ces organisations aident à la transformation du format de préoccupation de ces acteurs - lorsqu'il est familier en un format requis par le gouvernement par le standard, notamment par les 
procédures prévues de plaintes. En dépit des réductions de l'objectif, l'assemblage de ces différentes ONG fait place à une large gamme de formats de connaissance et d'évaluation, depuis la forme universelle de droits fondamentaux ou du consentement éclairé des peuples jusqu'au format des usages familiers bienfaisants. Certaines ONG ont conçu des dispositifs ouverts à cette large gamme, cherchant parfois à faciliter autant les critiques internes que des critiques externes exposées aux marges de la RSPO. Ces organisations procèdent à des enquêtes auprès des communautés locales concernées (Colchester \& Chao 2013). Réalisées souvent par des membres de ces ONG formés à l'anthropologie, les investigations épousent des formats de connaissances et d'évaluation qui s'écartent des formats évaluatifs liés aux objectifs des bonnes pratiques et aux savoirs experts industriels.

Le rapport "Conflict or consent?" (voir supra $\$ 2.3$ ) est révélateur de ce travail en commun d'une chaine d'ONG. Au-delà de la visibilité donnée à des plaintes de communautés locales à travers les études de cas, ce rapport a contribué à faire avancer, dans les jours et mois suivants sa parution, des dossiers plus généraux. Il a facilité, nous l'avons souligné, l'adoption d'une nouvelle résolution par l'Assemblée Générale de la RSPO sur l'indépendance du jury statuant sur les plaintes (résolution 6f). Il a conduit également au renforcement de la légitimité du concept de FPIC par le développement de manuels (guidelines pour la mise en œuvre du FPIC). Il a aussi abouti à la réalisation d'une étude sur les organisations «intermédiaires » à même de faciliter l'accès des communautés locales au mécanisme des plaintes et, enfin, à la révision des procédures du mécanisme des plaintes lui-même. Cette révision fut actée dans un contexte de critiques s'étendant aux formats de preuves, ceux-ci limitant l'accès des communautés locales au mécanisme de plainte.

63 Au-delà d'une coalition efficace et stratégique d'ONG, qui vient soutenir une participation plus critique à la table ronde, c'est l'articulation d'ONG, dans une chaîne complexe de compétences variées, qui permet à ce rapport d'avoir un tel impact. Considéré par les participants de la RSPO comme "bien documenté ", le rapport s'appuie sur une variété de formats d'information et de connaissance, qui permettent une remontée de la plainte jusqu'au niveau transnational en évitant des réductions sévères. Sont ainsi mobilisés une connaissance très fine des textes juridiques et législatifs, des documents officiels et des procédures d'opération des plantations. Contrairement aux réductions expertes propres à l'audit, des preuves sont aussi apportées à partir de témoignages oraux recueillis sur le terrain, de photos prises sur les sites pour mettre en évidence les conditions de vie locales et les attachements (photos de sites sur lesquels des tombes ont été détruites par exemple), des témoignages écrits anciens (datés du XIXème siècle, par exemple signés du sultan de Sambas en écriture arabe), et toute une série d'éléments probants au niveau local pour les personnes résidentes.

64 Le rapport "Conflict or consent?" tente ainsi de faire remonter une plus grande diversité de maux causés par l'extension des sociétés de plantation: dégradation des moyens d'existence, déracinement de personnes et enclavement de villages, la perte de biens familiers, ou encore de terres - constitutives des personnes - où sont présents les tombes des ancêtres, des arbres sacrés, et les marques d'une transmission générationnelle ouverte à des usages familiers et spirituels. 


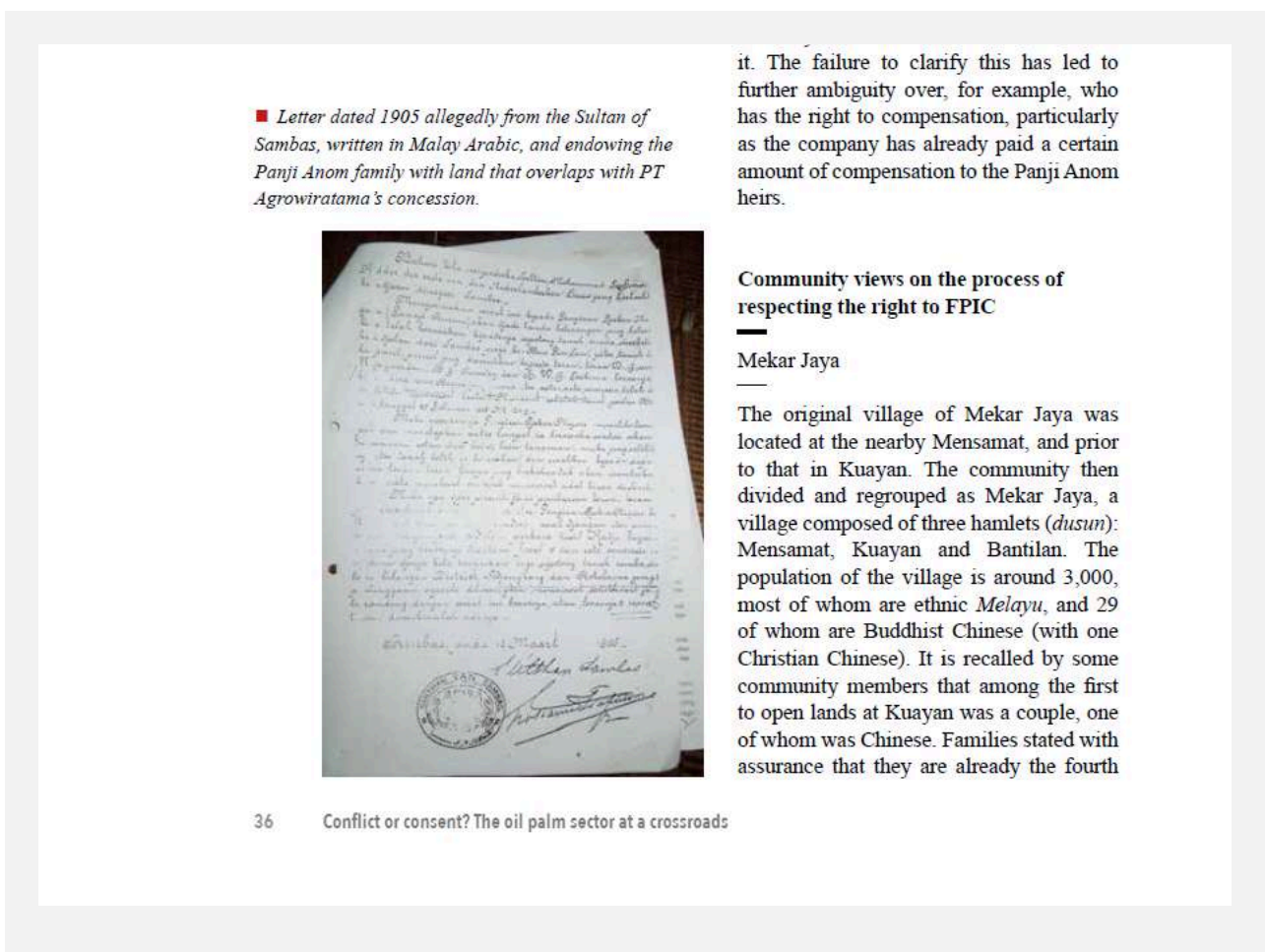

Photo 1 : Usage d'une lettre datée de 1905, du Sultan de Sambas, écrite en lettres arabes Malay (Colchester and Chao, 2013).

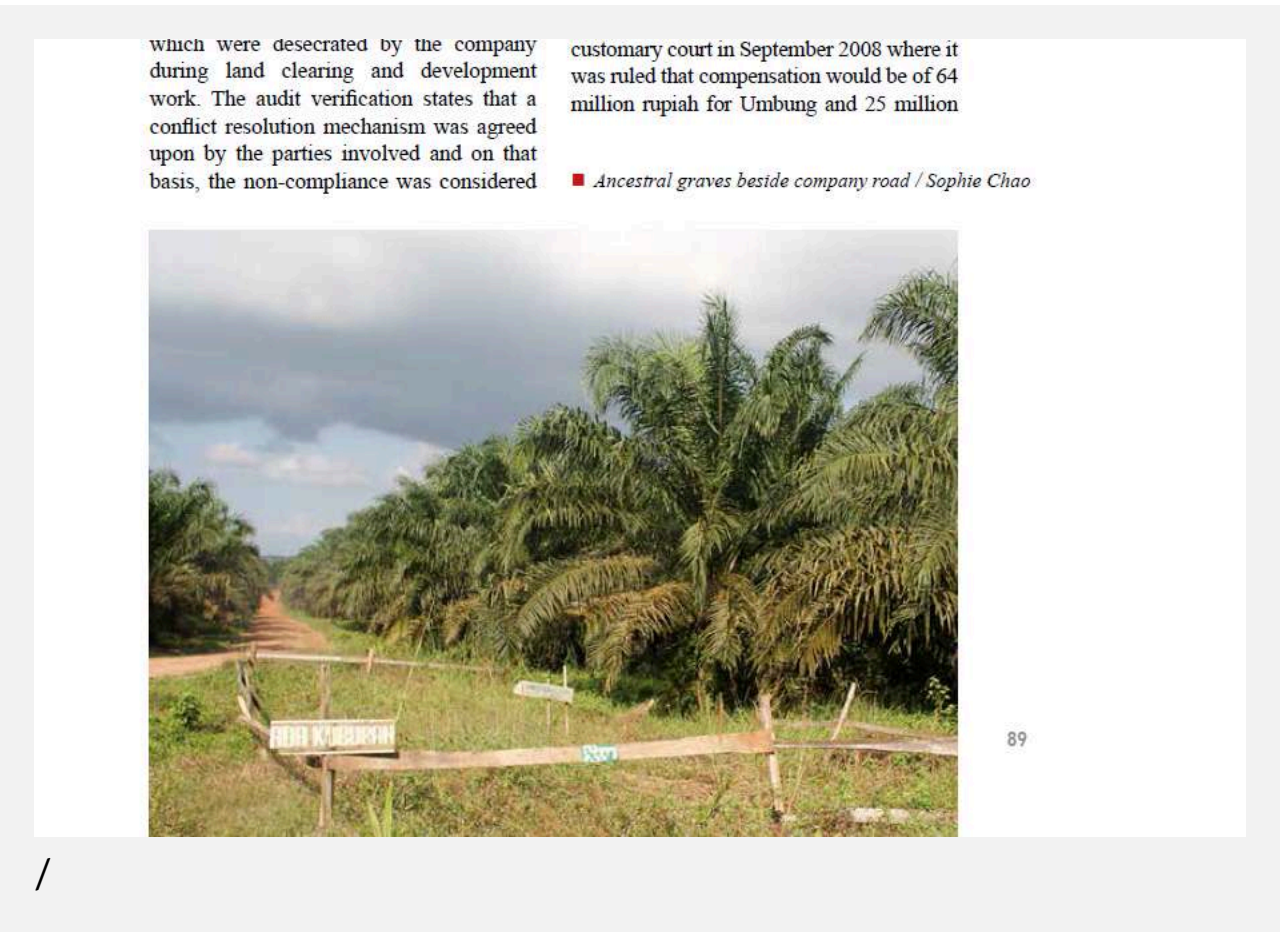

Photo 2: Le rapport explique que ces tombes ont été détruites pour construire une route dans la plantation. La route a ensuite été déviée et l'emplacement signalé par un panneau (Colchester and Chao, 2013). 


\section{PT Permata Hijau Pasaman I and the Kapa and Sasak peoples of Pasaman Barat, West Sumatra \\ 4}

Patrick Anderson, Fatilda Hasibuan, Asep Yunan Firdaus, Afrizal, Zulkifli and Nurul Firmansyah

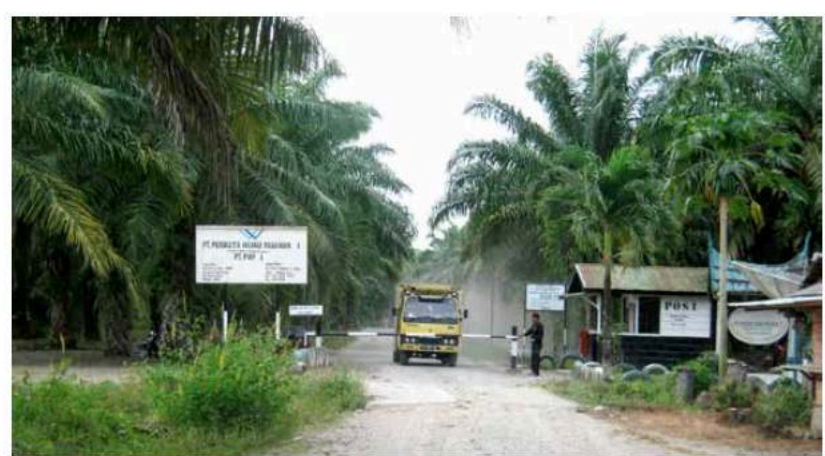

Introduction

PT Permata Hijau Pasaman I ${ }^{1}$ (PT PHP I)

is a member of the Wilmar group, which
- Gateway to PT PHP I concession. The Nagari Kapa village of Talao Pagang is surrounded by the concession and villagers must pass by this gate to enter or leave their village.
3 : Porte d'entrée dans la concession ; le village est entouré par la concession, et les habitants doivent passer par cette porte pour entrer ou quitter leur village (Colchester and Chao, 2013).

Letai et le complément apportés par ces ONG au gouvernement par le standard RSPO se manifestent aussi dans l'aide au cheminement des plaintes dans les deux circuits mentionnées plus haut: «RSPO Complaints System» et «Dispute Settlement Facility »(médiation). Là encore, la disposition d'une variété d'ONG plus ou moins locales et liées les unes aux autres contribue à ce que les voix des minorités soient entendues et qu'il soit fait quelque place dans le différend aux formats écartés par l'objectif, celui d'attachements personnels et celui de biens fondamentaux mis en avant par un sens de l'injustice (Cheyns 2014). Compte tenu du cadrage technique imposé dans les tables rondes, la participation d'ONG locales et nationales revêt une importance majeure dans la remontée des préoccupations locales. Les ONG locales jouent un rôle clé pour faire remonter certaines formes de preuves, qui font sens localement mais qui sont difficilement compréhensibles en l'état par une communauté internationale étrangère au terrain. Elles favorisent pour cela leur «transformation ». Des repères familiers qui font sens localement, pour établir une frontière par exemple, sont le plus souvent invisibles aux auditeurs ou aux étrangers des lieux. C'est le cas par exemple d'arbres, du fait de leur âge et de leur histoire, parfois gravés d'un nom ou d'un code. Si ces repères font sens localement, pour les populations résidentes, celles-ci font face à une difficulté de taille : que ces formes d'évidence soient reconnues et mêmes vues, par exemple par un auditeur, qui reçoit de l'autre partie (la société de plantation avec qui les résidents sont en conflit), un monceau de documents techniques, procéduraux et formels (SilvaCastañeda, 2012b).

Le transport de ces formes de preuves dans une instance supranationale telle que la RSPO constitue également une difficulté de taille. Les plaintes des communautés locales déposées à la table ronde sont reçues par son Secrétariat exécutif, puis traitées par un «panel d'experts» (nommés, et membres de la table ronde). Ces plaintes doivent être 
mises en forme, d'abord par les plaignants, afin de satisfaire les exigences de l'évaluation de la "recevabilité de la plainte ", puis par le Secrétariat exécutif, qui rédige un résumé du cas à destination du panel d'experts. Ces derniers statuent uniquement sur la base des pièces transmises par le Secrétariat. Ils ne se déplacent pas sur le terrain ni n'entendent les parties, l'identité des experts traitant le cas devant rester anonyme.

70 Cette distance des experts s'oppose au désir des communautés locales affectées, qui souhaitent au contraire que ceux-ci se déplacent « et viennent voir sur le terrain ». Au cœur de cette demande, les communautés locales expriment un souci majeur. Les experts devraient aller sur les lieux pour accéder aux formats de preuve non transportables ni facilement transformables, ressentir la sincérité de leur plainte qui est souvent mise en cause par la compagnie accusée, comprendre les dommages qu'elles subissent ${ }^{30}$. Colchester mentionne lui aussi ce désir des ONG que les experts du panel ou de RSPO se déplacent sur le terrain, plutôt que de statuer ou évaluer les cas sur la base de rapports et de courriers lettre-réponse entre RSPO et les parties, qu'il qualifie de «e-mail ping pong " (voir son entretien dans le volume).

71 A défaut de déplacement du panel et du secrétariat sur le terrain, les ONG locales jouent un rôle clé dans la mise en forme d'éléments de preuve, en favorisant leur transformation. Cette transformation est cruciale pour faire remonter une plainte sur l'arène transnationale et en particulier au niveau du mécanisme de traitement des plaintes. Ces ONG interviennent par exemple dans la transformation de récits autour du conflit en "éléments chronologiques", plus adaptés aux demandes du Complaint System.

Elles interviennent aussi dans la transformation de repères anciens et familiers en cartes, grâce à des exercices de cartographie participative qui accordent de la valeur aux témoignages locaux et collectifs. De cette manière, des repères familiers reconnus sur place par un ancien du village peuvent être transposés en coordonnées GPS, et les cartes transportées vers une plus large audience: "With these participatory maps we can convert the local knowledge, the local landmarks (as rivers, trees) into boundaries that can be understandable by modern practices »(ONG, Jambi, 2013; voir aussi : Thévenot 2019). La cartographie participative est aussi utilisée pour faire valoir une variété des usages (photo 4). 


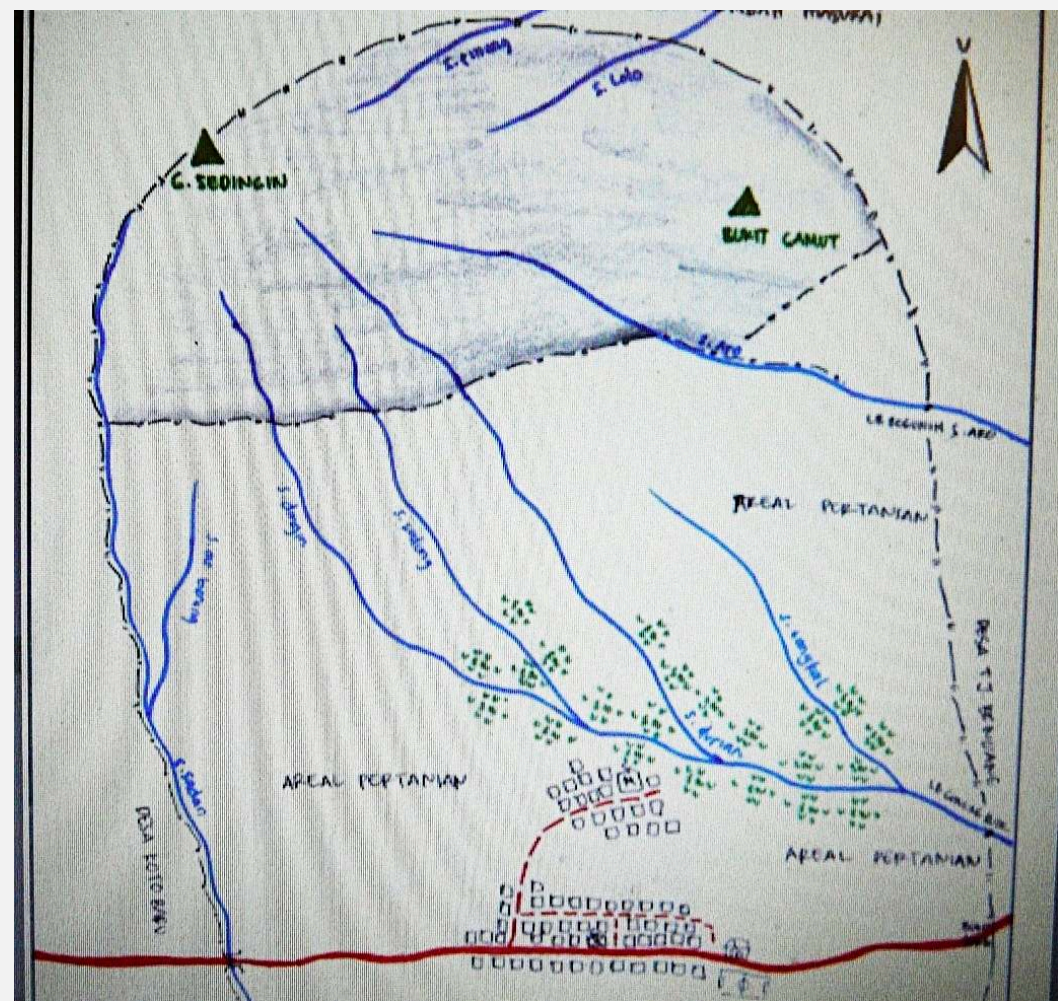

Photo 4. Exemple de carte indiquant la variété des usages des lieux, pour les faire valoir contre l'implantation de palmiers à huile

La capacité des ONG locales et nationales à utiliser différents formats de preuves est liée à une composition très diverse de leurs membres et, pour nombre d'entre eux, à leur proximité avec les communautés locales (certains partageant leur quotidien). Elle est également le fait d'un travail en réseau, articulant des ONG proches du terrain et des ONG nationales, elles-mêmes en relation avec des ONG internationales et la RSPO (voir aussi Köhne 2014).

\section{Conclusion : un pied dedans, un pied dehors}

Dans un esprit managérial prompt à mettre en avant des innovations, le gouvernement par les standards de certification se présente dans ses textes prescriptifs comme un dispositif autonome original. De fait, nous avons mis en relief ses traits distinctifs grâce à notre perspective comparative. Cependant l'analyse précise de son fonctionnement à partir de situations critiques et des évolutions qu'elles entraînent, de même que celle d'oppressions qui vont au-delà des asymétries de pouvoir les plus criantes, ont montré qu'il fallait replacer les outils normatifs de ce gouvernement dans une espace beaucoup plus ample de régimes normatifs avec lesquels ils se trouvent explicitement ou non imbriqués.

La mise en évidence des limites structurelles du gouvernement par les standards et objectifs dans la prise en compte des biens dont se préoccupent les plus défavorisés peut conduire à se demander si la participation au demeurant très coûteuse de ces derniers au dispositif a ses raisons d'être. De fait, certaines organisations paysannes telles que Via Campesina ont fait le choix de rester à l'extérieur pour le critiquer. Le 
vis-à-vis de ces populations défavorisées, en Indonésie et plus encore en Malaise, est tel qu'il fait obstacle à l'expression de la critique, qu'il s'agisse des organes de gouvernement nationaux, du système judiciaire en acte ou des compagnies de la filière de l'huile de palme. Face à l'irresponsabilité civique des entreprises et à la relative faiblesse des Etats-nations dans leurs relations avec les multinationales (sur ces questions, voir : Leader 2018), RSPO est un dispositif où les agents les plus " petits » - au regard de ces acteurs publics et privés auxquels ils sont quotidiennement confrontés trouvent des lieux et des soutiens connexes d'ONG pour faire venir des exigences de responsabilité. Dans un des cas que nous avons suivis en Indonésie, la tactique développée pour défendre les plus défavorisés consiste à conjuguer la participation à la RSPO à des modalités toutes différentes, critiques, de constitution et de défense des petits planteurs par un syndicat propre et par des manifestations publiques, ou encore par des efforts de longue durée pour des réformes de la réglementation nationale.

\section{BIBLIOGRAPHIE}

Blok, A., 2013, "Pragmatic sociology as political ecology: on the many worths of nature(s)", European Journal of Social Theory 16(4): 492-510.

Boltanski, L., Thévenot, L., 1991, De la justification. Les économies de la grandeur, Paris, Gallimard.

Busch, L., 2000, "The Moral Economy of Grades and Standards", Journal of Rural Studies, 16(3): 273-283.

Busch, L., 2011, Standards. Recipes for reality, Cambridge, MIT Press.

Colchester, M., 1982, "Les Yanomami, sont-ils libres ? Les Utopies Amazoniennes, une critique. A look at French Anarchist Anthropology". Journal of the Anthropological Society of Oxford 13 (2): 147-64.

Colchester, M., 2002, "Indigenous rights and the collective conscious", Anthropology Today, 18(1), pp. 1-3.

Colchester, M., 2006, "Facing Kuper: Indigenous Peoples, Human Rights and Democratic Development", talk to the London School of Economics organised by the LSE Students Union Anthropology Society and DESTIN society, 13 February.

Colchester, M., Chao, S. (eds), 2013, Conflict or Consent, Forest Peoples Programme, Sawit Watch and TUK INDONESIA.

Charles, J., 2012, "Comment la cartographie méconnaît les habitants. Le formatage de la participation dans une commune belge", Participations. Revue de sciences sociale sur la démocratie et la citoyenneté $\mathrm{n}^{\circ} 3$, pp. 155-178

Charles, J., 2015, Une participation sans condition? Une sociologie des épreuves de l'engouement participatif, Paris, Desclée de Brouwer (préface de L. Thévenot). 
Cheyns, E., 2011, "Multi-stakeholder initiatives for sustainable agriculture: limits of the 'Inclusiveness' paradigm", in Ponte, Stefano, Gibbon, Peter and Vestergaard Jakob (eds.), Governing through Standards. Origins, Drivers and Limitations, London, Palgrave, pp. 210-235.

Cheyns, E., 2014, "Making 'minority voices' heard in transnational roundtables: The role of local NGOs in reintroducing justice and attachments", Agriculture and Human Values, vol $31 \mathrm{n}^{\circ} 3$, pp. 439-453.

Cheyns E. and Riisgaard L., 2014, "Introduction to the symposium". The exercise of power through multi-stakeholder initiatives for sustainable agriculture and its inclusion and exclusion outcomes, Agriculture and Human Values, vol 31 n 3, pp. 409-423.

Cheyns E., Silva-Castañeda L., Aubert P.-M., 2018, "Missing the forest for the data? Conflicting valuations of the forest and cultivable lands", Land Use Policy (forthcoming).

Colchester, M., 1982, "Les Yanomami, sont-ils libres ? Les Utopies Amazoniennes, une critique. A look at French Anarchist Anthropology". Journal of the Anthropological Society of Oxford 13 (2): 147-64.

Colchester, M., 2002, "Indigenous rights and the collective conscious", Anthropology Today, 18(1), pp. 1-3.

Colchester, M., 2006, "Facing Kuper: Indigenous Peoples, Human Rights and Democratic Development", talk to the London School of Economics organised by the LSE Students Union Anthropology Society and DESTIN society, 13 February.

Colchester, M., 2011, "Divers Paths to Justice: Legal pluralism and the rights of indigenous peoples in Southeast Asia - an introduction", in Colchester, Marcus, Chao, Sophie (eds), Divers Paths to Justice: Legal pluralism and the rights of indigenous peoples in Southeast Asia, published by Forest Peoples Programme (FPP) and Asia Indigenous Peoples, pp. 17-39.

Dworkin, G., 1988, The Theory and Practice of Autonomy, Cambridge, Cambridge University Press.

Engle K., 2011, "On Fragile Architecture: The UN Declaration on the Rights of Indigenous Peoples in the Context of Human Rights", The European Journal of International Law 22(1): 141-163.

Eranti, V., 2018, "Engagements, grammars, and the public: from the liberal grammar to individual interests", European Journal of Cultural and Political Sociology, 5(1-2) Special issue on "Politics of Engagements", pp. 42-65.

Faden, R. R. \& Beauchamp, T. L., 1986, A History and Theory of Informed Consent, Oxford, OUP.

Fan, R., 2015, Family-Oriented Informed Consent: East Asian and American Perspectives, Cham, Springer.

Köhne, M. (2014). Multi-stakeholder initiative governance as assemblage: Roundtable on Sustainable Palm Oil as a political resource in land conflicts related to oil palm plantations. Agriculture and human values, 31(3), 469-480.

Kuper, A., 1999, Culture. The Anthropologists' Account, Cambridge, MA-London, Harvard University Press.

Lafaye, C., Thévenot, L., 1993, "Une justification écologique ? Conflits dans l'aménagement de la nature", Revue Française de Sociologie, vol. 34, n 4, oct.-déc., pp. 495-524.

Leader, S., 2000, "Three faces of justice and the management of change", Modern Law Review, vol. 6, january, pp. 55-83. 
Leader, S., 2005, "Two ways of linking economic activity to human rights», International Social Science Journal, 2005, $\mathrm{n}^{\circ}$ 185, pp. 541-550.

Leader, S., 2009, "The Collateral Protection of Rights in a Global Economy", New York Law School Law Review, 53(4), pp. 805-814.

Leader, S., 2010, "Labour Rights in the World Economy", in Lyon-Caen A. et Perulli A. (dir.), 2010, Valutare il diritto del lavoro. Evaluer le droit du travail. Evaluate labour law, Padova, Cedam, pp. 113-122.

Leader, S., 2018, "Institutional Variety, Civil Society and Basic Rights", in Jeammaud, A., Le Friant, Ma., Lokiec, P., Wolmark C. (eds.), Le droit sans frontières, Paris Dalloz.

Lénine, 1973 [1914], Du droit des nations à disposer d'elles-mêmes, Paris Ed. Sociales, Moscou Ed. du Progrès.

Li, T. M., 2015. After the land grab : Infrastructural violence and Indonesia's oil palm zone. $17 \mathrm{p}$. BICAS, MOISAIC, LDPI, RCSD, Transnational Institute.

Lomelino P. J. , 2015, Community, Autonomy and Informed Consent: Revisiting the Philosophical Foundations for Informed Consent, Cambridge Scholars Publishing.

Luhtakallio, E. \& Thévenot, L., 2018, "Politics of Engagement in an Age of Differing Voices" (introduction to the special issue) European Journal of Cultural and Political Sociology 5(1-2), 1-11.

Lyon-Caen, A., Champeil-Desplats, V., (eds.), 2001, Services publics et droits fondamentaux dans la construction européenne, Paris, Dalloz.

Lyon-Caen A., Lokiec P. (eds.), 2005, Droits fondamentaux et droit social, Paris, Dalloz.

Lyon-Caen A. et Perulli A. (dir.), 2010, Valutare il diritto del lavoro. Evaluer le droit du travail. Evaluate labour law, Padova, Cedam.

Manila Declaration of The International Conference. On Conflict Resolution, Peace Building, Sustainable Development And Indigenous Peoples, organized and convened by Tebtebba Foundation (Indigenous Peoples' International Centre For Policy Research and Education) in Metro Manila, Philippines on December 6 - 8, 2000.

Moody, M., Thévenot, L. 2000, "Comparing Models of Strategy, Interests, and the Public Good in French and American Environmental Disputes", in Lamont Michèle and Thévenot Laurent (eds.), Rethinking Comparative Cultural Sociology: Repertoires of Evaluation in France and the United States, Cambridge, Cambridge University Press, pp. 273-306.

Pattaroni, L., 2005, « Le care est-il institutionnalisable ? Quand la « politique du care » émousse son éthique ", in Paperman, Patricia et Laugier, Sandra (eds.), Le souci des autres, (Raisons Pratiques 16) Paris, Ed. de l'EHESS, pp. 177-200.

Ponte, St., Gibbon, P. and Vestergaard J. (eds.), 2011, Governing through Standards. Origins, Drivers and Limitations, Palgrave Macmillan.

Ponte, S. and Cheyns, E., 2013, "Voluntary standards, expert knowledge and the governance of sustainability networks", Global Networks, 13(4), 459-477.

Power M., 1997, The Audit Society. Rituals of Verification, Oxford, Oxford University Press.

Raymond, M. \& Denardis, L., 2015, "Multistakeholderism: anatomy of an inchoate global institution", International Theory 7:3, 572-616.

Richard-Ferroudji, A., 2011, "Limites du modèle délibératif : composer avec différents formats de participation", Politix, 24(96) : 161-181. 
Richards, A., Kuper, A. (eds), 1971, Councils in action, Cambridge, Mass., Cambridge University Press.

Silva Castaneda, L. 2012a. Les dispositifs de certification à l'épreuve des conflits fonciers : le cas de la table ronde sur l'huile de palme durable (Doctoral dissertation, UCL-Université Catholique de Louvain).

Silva-Castañeda, L. 2012b. A forest of evidence: third-party certification and multiple forms of proof - a case study on oil palm plantations in Indonesia. Agriculture and human values 29: 361-370.

Silva-Castañeda, L. (2015). What Kind of Space? Multi-stakeholder Initiatives and the Protection of Land Rights. International Journal of Sociology of Agriculture \& Food, 22(2).

Thévenot, L., 1984, "Rules and implements: investment in forms", Social Science Information, vol. $23, \mathrm{n}^{\circ} 1$, pp. 1-45.

Thévenot, L., 1995, "Des marchés aux normes", in Allaire, G., Boyer, R. (eds.), La grande transformation de l'agriculture : lectures conventionnalistes et régulationnistes, Paris, INRA-Economica, pp. 33-51.

Thévenot, L., 1997, "Un gouvernement par les normes ; pratiques et politiques des formats d'information", in Conein, B. et Thévenot, L. (dir.), Cognition et information en société, Paris, Ed. de l'EHESS (Raisons Pratiques 8), pp. 205-241.

Thévenot, L., 2008, "Sacrifices et bénéfices de l'individu dans un espace public libéral", Cahier d'éthique sociale et politique, $\mathrm{n}^{\circ} 5$, pp. 68-79.

Thévenot, L., 2014, "Voicing concern and difference. From public spaces to common-places", European Journal of Cultural and Political Sociology, 1(1) 7-34.

Thévenot, L., 2015a, "Making commonality in the plural, on the basis of binding engagements", in Dumouchel Paul and Gotoh Reiko (eds.), Social Bonds as Freedom: Revising the Dichotomy of the Universal and the Particular, New York, Berghahn, pp. 82-108.

Thévenot, L., 2015b, "Autorités à l'épreuve de la critique. Jusqu'aux oppressions du 'gouvernement par l'objectif" in Frère Bruno (sous la dir.) Le tournant de la théorie critique, Paris, Desclée de Brouwer, pp. 216-235.

Thévenot, L., 2015c, "Certifying the world. Power infrastructures and practices in economies of conventional forms", in Aspers, Patrick and Nigel Dodd (eds.), Re-Imagining Economic Sociology. Oxford: Oxford University Press, pp. 195-223.

Thévenot, L., 2019, "A new calculable global world in the making: governing through transnational certification standards", in Mennicken, Andrea \& Salais, Robert (eds.), The New Politics of Numbers: Quantification, Administrative Capacity and Democracy, Palgrave (forthcoming).

Valor C. \& A. Merino de Diego A., 2009 « Relationship of business and NGOs: an empirical analysis of strategies and mediators of their private relationship », Business Ethics: A European Review, 18(2), 110-126.

Weindling, P. J., 2004, Nazi Medicine and the Nuremberg Trials: From Medical Warcrimes to Informed Consent, New York, Palgrave.

\section{NOTES}

1. La désignation des biens communs en italique renvoie à la pluralité des ordres de « grandeur» (Boltanski et Thévenot 1991). 
2. Cette série d'enquêtes a porté sur les modalités d'exclusion de voix, en raison des formats de participation et de connaissance mobilisés dans le processus de développement du standard (Cheyns, 2011, Cheyns et Riisgaard 2014, Ponte et Cheyns 2013) et dans sa mise en œuvre (SilvaCastaneda, 2012a, 2012b et 2015). Elle s'est prolongée par une analyse des modalités d'action d'une chaine d'ONG pour faciliter l'inclusion des "personnes affectées" au sein du dispositif (Barbereau, 2010, Cheyns, 2014), ainsi que leur accès aux mécanismes des plaintes de RSPO, avec L. Thévenot en 2013 (Thévenot 2019) puis avec C. Dumont en 2015. Nous remercions les personnes qui ont facilité ces dernières enquêtes, les ONG Sawit Watch, Walhi, Forest People Programme et le Secrétariat RSPO.

3. La référence au gouvernement «multistakeholder » a été beaucoup utilisée dans le domaine de l'Internet. Une analyse plus précise a montré que l'inégalité de poids des acteurs différenciés par «stakes » (state, firm, civil society) contrevenait au modèle (Raymond \& Denardis 2015).

4. En droit français, le terme "consentement" traduit correctement l'anglais " consent». Cependant, la catégorie juridique du consentement, qui désigne le fait de se prononcer en faveur d'un acte juridique, d'une convention ou d'un contrat, repose sur la doctrine de l'autonomie de la volonté qui est précisément celle dont nous discutons en première partie les fondements normatifs et les limites. C'est pourquoi nous avons retenu dans le titre de cette section une notion moins spécifiée par cette doctrine, plus large dans ses modalités pratiques et fondements normatifs, que nous avons dénommée « accord ». Notre utilisation du terme est moins spécifiée que ses usages juridiques désignant en droit.

5. Qui lui valu en 2001 la 'Lucy Mair Medal for Applied Anthropology' décernée par le Royal Anthropological Institute.

6. Comme notre chapitre tâche de le montrer, la sociologie politique et morale des engagements offre d'autres ressources critiques qui permettent aussi d'identifier des limites de la critique féministe. Elles tiennent au court-circuit opéré entre la reconnaissance du "care" et son grandissement politique public. Sur ces limites, voir Pattaroni 2005.

7. Li (2015) parle de « violence infrastructurelle », liée à la concentration des marchés (monopole des concessions pour l'achat des régimes).

8. La présentation schématique qui suit ne doit cependant pas faire oublier que les deux systèmes se trouvent de fait imbriqués.

9. La révision du standard est prévue tous les 5 ans, selon les principes d'ISEAL; elle est de nouveau en cours en 2018.

10. Voir les précisions sur cette question apportées par l'entretien avec Marcus Colchester publié dans ce volume, et notamment les tensions issues de la rencontre entre le droit national et l'application du principe FPIC.

11. Cette prise en compte est confirmée dans le principe 7.5. (et 7.6.) concernant l'établissement de nouvelles plantations, où la mention de FPIC est clarifiée avec l'ajout : « les populations locales concernées comprennent leur droit de dire «non » aux opérations prévues sur leurs terres (...) ». Il est fait référence, via une annexe, à la convention ILO 169 (relative aux peuples indigènes et tribaux) et la déclaration des Nations unies sur les droits des peuples autochtones.

12. "6.1 "Les activités de gestion de la plantation et de l'usine, y compris la replantation, qui ont un impact social sont identifiées de façon participative, et des programmes d'atténuation des impacts négatifs et de promotion des effets positifs sont développés, mis en œuvre et suivis afin de démontrer la poursuite d'une amélioration continue ». 6.1.3 précise que ces "plans » seront consignés et accompagnés de calendriers d'exécution ( documented and timetabled»), établis en consultation avec les parties affectées (" in consultation with the affected parties»); 6.1.4 qu'ils seront révisés au moins tous les deux ans.

13. SIRIM QAS international, 2103, "RSPO surveillance assessment report», Sime darby plantation sdn.bhd. rajawali certification unit (sou 32), Bintulu district, Sarawak, Malaysia 
assessment date: 5th to 9th November 2012, http://www.rspo.org/sites/default/files/ Audit_Report-Rajawali_SOU_-_SA_2012-Final-130905-signed-11.pdf

14. Déclaration de l'OIT relative aux principes et droits fondamentaux au travail, 1998.

15. Conventions 87, 98 et 138 .

16. Les deux derniers principes sont quant à eux plus spécifiques et visent les nouvelles plantations (7. » Développement responsable de nouvelles plantations » avec l'ajout 7.8 sur les gaz à effet de serre) ainsi que leur amélioration continuelle (8. » Engagement envers une amélioration continue des principaux domaines d'activité »).

17. Voir partie 3 et les précisions apportées par Marcus Colchester dans son entretien, à propos des limites de l'audit - en particulier de cette approche à partir des objectifs - et des contraintes qu'elle pose sur le respect et la considération du FPIC.

18. La « Dutch coalition soya » associe des ONG membres de la table ronde sur le soja responsable (RTRS), telles que WWF, et des ONG extérieures (telles que Friends of the Earth et, pendant les premières années, Greenpeace, 2 ONG extérieures à la RTRS) dans l'élaboration de stratégies communes sur la filière soja.

19. Greenpeace Pays-Bas, 2008. United Plantations certified despite gross violation of RSPO Standards. 13 p. https://www.greenpeace.org/archive-international/Global/international/planet-2/report/ 2008/11/united-plantations-certified-d.pdf

20. M. Colchester \& S. Chao (eds), 2013. Conflict or Consent ? The oil palm sector at a crossroads. Forest Peoples Programme, Sawit Watch and TUK INDONESIA, 417 p.

21. Ce rapport documente 16 cas de conflits dans des pays d'Afrique, en Malaisie et en Indonésie.

22. Grassroots \& EIA, 2015. Who watches the watchmen? Auditors and the breakdown of oversight in the RSPO. $24 \mathrm{p}$.

23. L'agence fédérale malaysienne de développement FELDA a lancé un grand nombre de compagnies privées. La plus importante, FELDA Global Ventures Holdings (FGV), est considérée comme opérant la plus grande plantation mondiale avec 811,140 hectares de palmiers à huile.

24. Conglomérat malaisien fondé en 1969 (230 000 ha de plantations en Malaisie et en Indonésie).

25. Ce plan suppose qu'IOI rende compte de l'avancement du plan d'action approuvé de façon trimestrielle, avant de se soumettre à une nouvelle vérification indépendance au terme d'une année.

26. AIDEnvironment. "Talking Responsibility. Review of IOI Group's majority owned active subsidiaries in Ketapang District ». https://rspo.org/files/download/032ad0b044cb2da

27. https://www.greenpeace.org/usa/news/international-ngos-condemn-ioi-groups-plan-divestconflict-palm-oil-plantation/

28. A l'inverse, la peine de mort peut être jugée excessive au regard des coutumes.

29. La première étape de la sociologie ici mise en œuvre était consacrée aux « investissements de forme » qui facilitent l'accord par l'équipement de formes conventionnelles d'équivalence, et avait conduit à puiser dans une littérature d'anthropologues sur les conseils tribaux (Thévenot 1984). L'ouvrage collectif de référence consulté et cité était consacré aux «Conseils en action » (Richards \& Kuper 1971) et codirigé par Audrey Richards et le même Adam Kuper que Colchester allait devoir affronter 35 ans plus tard à Londres...

30. Sur la signification profonde, en termes de format de preuve, de cette demande que les interlocuteurs « viennent sur le terrain », voir aussi : Richard-Ferroudji 2011. 


\section{RÉSUMÉS}

Parmi les instruments normatifs contemporains de régulation, les standards privés et volontaires relèvent d'un véritable mode nouveau de gouvernement en quête de légitimité. A partir du standard RSPO (Roundtable on Sustainable Palm Oil), nous analysons le modèle politique normatif sous-jacent aux initiatives dites multi-stakeholders. En s'appuyant sur une composition libérale du commun, ces initiatives remodèlent et transforment des éléments de droits internationaux tels que la notion de consentement des peuples (Free Prior and Informed Consent) que des ONG ont introduite dans le standard. Nous comparons le gouvernement RSPO » au droit national et au fonctionnement d'un Etat quant à ses fonctions légitimes et principes idéaux. Nous comparons en particulier les fonctions de police et de justice et leurs modalités d'expression. Enfin, nous abordons les questions soulevées par le pluralisme des modes de normativité et d'évaluation confrontés dans ce gouvernement. Les préoccupations des parties prenantes reconnues comme les plus vulnérables ("petits planteurs", "communautés locales") doivent être transformées (et réduites) pour être recevables dans le standard RSPO et son gouvernement, qu'il s'agisse d'expressions de leur accord pour l'usage de terres par des entreprises privées, de demandes ou de plaintes qu'elles déposent.

Among contemporary normative instruments of regulation, private and voluntary standards are a new mode of government in search of legitimacy. By considering the RSPO standard (Roundtable on Sustainable Palm Oil), we analyze the normative political model underlying the so-called "multi-stakeholder" initiatives. Relying on a liberal composition of commonality and difference, these initiatives reshape elements of international human rights such as the notion of people's consent (Free Prior and Informed Consent) that NGOs introduced in the standard. We compare the "RSPO government" to national law, and the functioning of a state with respect to its legitimate functions and ideal principles, in particular, police and justice functions. Finally, we address the issues raised by the plurality of modes of normativity and evaluation in the RSPO government. The most vulnerable stakeholders' ("smallholders'", "local communities") concerns have to be reshaped (and reduced) into formats that are accepted by the RSPO standard and government, when expressing their agreement for the use of their lands by corporations, or filing claims or complaints.

\section{INDEX}

Mots-clés : droit, standard, normativité, sociologie pragmatique, communautés locales, consentement, plainte, multi-parties prenantes.

Keywords : law, standard, normativity, pragmatic sociology, local communities, free consent, complaint, multistakeholder.

\section{AUTEURS}

\section{EMMANUELLE CHEYNS}

Emmanuelle Cheyns est chercheure en sociologie au Cirad. Elle est membre de l'UMR Moisa et mène des recherches sur les standards volontaires de durabilité. 


\section{LAURENT THÉVENOT}

Laurent Thévenot est Directeur d'études honoraire à l'Ecole des Hautes Etudes en Sciences Sociales, Paris, Centre Georg Simmel (EHESS-CNRS). 